quatrième série-tome 43 fascicule 1 janvier-février 2010

$$
\begin{aligned}
& \text { ANNALES } \\
& \text { SCIENTIFIQUES } \\
& \text { de } \\
& \text { L'ECOLE } \\
& \text { NORMALE } \\
& \text { SUPÉRIEURE }
\end{aligned}
$$

Thierry GOUDON \& Alexis VASSEUR

Regularity analysis for systems of reaction-diffusion equations 


\title{
REGULARITY ANALYSIS FOR SYSTEMS OF REACTION-DIFFUSION EQUATIONS
}

\author{
By Thierry GOUDON and Alexis VASSEUR
}

\begin{abstract}
This paper is devoted to the study of the regularity of solutions to some systems of reaction-diffusion equations. In particular, we show the global boundedness and regularity of the solutions in one and two dimensions. In addition, we discuss the Hausdorff dimension of the set of singularities in higher dimensions. Our approach is inspired by De Giorgi's method for elliptic regularity with rough coefficients. The proof uses the specific structure of the system to be considered and is not a mere adaptation of scalar techniques; in particular the natural entropy of the system plays a crucial role in the analysis.

RÉsumÉ. - Ce travail est consacré à l'étude de la régularité des solutions de certains systèmes d'équations de réaction-diffusion. En particulier, nous montrons que les solutions peuvent être bornées et régulières en dimensions un et deux alors qu'en dimensions supérieures nous discutons la dimension de Hausdorff de l'ensemble des points singuliers. L'approche proposée ici s'inspire de la méthode de De Giorgi pour étudier la régularité de problèmes elliptiques avec des coefficients discontinus. La preuve exploite la stucture spécifique des systèmes considérés et n'est pas une simple adaptation de techniques scalaires. L'entropie associée naturellement au système joue un rôle crucial dans cette analyse.
\end{abstract}

\section{Introduction}

This paper is devoted to the analysis of the following system of reaction-diffusion equations

$$
\left\{\begin{array}{l}
\partial_{t} a_{i}-\nabla \cdot\left(D_{i} \nabla a_{i}\right)=Q_{i}(a), \quad i \in\{1, \ldots, p\} \\
Q_{i}(a)=\left(\mu_{i}-\nu_{i}\right)\left(k_{\mathrm{f}} \prod_{j=1}^{p} a_{j}^{\nu_{j}}-k_{\mathrm{b}} \prod_{j=1}^{p} a_{j}^{\mu_{j}}\right), \\
a_{i \mid t=0}=a_{i}^{0} .
\end{array}\right.
$$

This work has been done while the first author was visiting ICES and the University of Texas at Austin. Thanks are addressed for their warm hospitality and support. We also thank J.-F. Coulombel who read preliminary versions and has suggested many improvements. The second author was partially supported by the NSF grant DMS0607053 . 
The equation holds for $t \geq 0$ and the space variable $x$ lies in $\Omega$ where

- either $\Omega=\mathbb{R}^{N}$,

- or $\Omega \subset \mathbb{R}^{N}$ is a bounded domain with smooth boundary and the system is completed by imposing the Neumann boundary condition

$$
D_{i} \nabla a_{i} \cdot \nu(x)_{\mid \partial \Omega}=0,
$$

where $\nu(x)$ stands for the outer normal vector at $x \in \partial \Omega$.

Throughout the paper, the symbol $\nabla$ denotes the gradient operator with respect to the space variable $x$ only. The matrices $D_{i}(x)$ are required to satisfy

$$
\begin{aligned}
& D_{i} \in\left(L^{\infty}(\Omega)\right)^{N \times N}, \\
& D_{i}(x) \xi \cdot \xi \geq \alpha|\xi|^{2}, \quad \alpha>0 \quad \text { for any } \xi \in \mathbb{R}^{N}, x \in \Omega .
\end{aligned}
$$

Let us comment this assumption:

- the analysis below is interesting when there are different diffusion matrices: assuming $D_{i}=D$, a common value, makes the problem easier;

- there is no regularity assumption on the coefficients;

- the standard uniform coercivity condition is assumed. The case of degenerate coefficients leads to specific difficulties which are beyond the scope of this paper.

Such a system is intended to describe e.g. the evolution of a chemical solution: the unknown $a_{i}$ stands for the density of the species labelled by $i \in\{1, \ldots, p\}$ within the solution. The right hand side of (1.1) follows from the mass action principle applied to the reversible reaction

$$
\sum_{i=1}^{p} \nu_{i} A_{i} \leftrightarrow \sum_{i=1}^{p} \mu_{i} A_{i},
$$

where the $\mu_{i}$ and $\nu_{i}$ 's - the so-called stoichiometric coefficients - are integers. The (positive) coefficients $k_{\mathrm{f}}$ and $k_{\mathrm{b}}$ are the rates corresponding to the forward and backward reactions, respectively. According to the physical interpretation, the unknowns are implicitly nonnegative quantities: $a_{i} \geq 0$. In fact, this property holds thanks to the structure of the system. Indeed, (1.1) can be written

$$
\partial_{t} a_{i}-\nabla \cdot\left(D_{i} \nabla a_{i}\right)+L_{i}(a) a_{i}=G_{i}(a)
$$

where the nonlinear functions $G_{i}$ and $L_{i}$ have the property: if the components $a_{k}$ of $a$ are non-negative then $G_{i}(a) \geq 0$ and $L_{i}(a) \geq 0$. Hence preservation of non-negativity, when starting from a non-negative initial data, can be considered among the a priori estimates of the problem (see appendix for more details). The main ingredients of our analysis rely on the following properties:

- The mass is conserved. The stoichiometric coefficients satisfy

$$
\text { There exists }\left(m_{1}, \ldots, m_{p}\right) \in \mathbb{N}^{p}, m_{i} \neq 0 \text {, such that } \sum_{i=1}^{p} m_{i} \mu_{i}=\sum_{i=1}^{p} m_{i} \nu_{i} .
$$

It implies the mass conservation

$$
\frac{\mathrm{d}}{\mathrm{d} t} \sum_{i=1}^{p} \int_{\Omega} m_{i} a_{i} \mathrm{~d} x=0 .
$$


- The entropy is dissipated. We set $K=k_{\mathrm{b}} / k_{\mathrm{f}}$, then

$$
\sum_{i=1}^{p} Q_{i}(a) \ln \left(a_{i} / K^{1 /\left(p\left(\mu_{i}-\nu_{i}\right)\right)}\right)=-k_{\mathrm{f}}\left(\prod_{i=1}^{p} a_{i}^{\mu_{i}}-K \prod_{i=1}^{p} a_{i}^{\nu_{i}}\right) \ln \left(\frac{\prod_{i=1}^{p} a_{i}^{\mu_{i}}}{K \prod_{i=1}^{p} a_{i}^{\nu_{i}}}\right) \leq 0 .
$$

In order to simplify the notations, and without loss of generality, we restrict ourselves to the case

$$
m_{i}=1, \quad k_{\mathrm{f}}=1=k_{\mathrm{b}} .
$$

A crucial role will be played by the quantity

$$
\bar{\mu}=\sum_{i=1}^{p} \mu_{i}=\sum_{i=1}^{p} \nu_{i}
$$

where the coefficients $\mu_{i}$ and $\nu_{i}$ are still integers.

In our study of such systems restrictions on the space dimension $N$ and the parameter $\bar{\mu}$ appear. One of the most interesting situations we are able to deal with is the following example corresponding to 4 species subject to the reactions

$$
A_{1}+A_{3} \leftrightarrow A_{2}+A_{4}
$$

It leads to

$$
Q_{i}(a)=(-1)^{i+1}\left(a_{2} a_{4}-a_{1} a_{3}\right) .
$$

We refer for a thorough introduction to the modeling issues and mathematical properties of such reaction diffusion systems to $[11,13,14,19,20,21,23,28,31]$. Information can also be found in the survey [6] with connection to coagulation-fragmentation models and in [24] for applications in biology. Let us also mention that (1.1) can be derived through hydrodynamic scaling from kinetic models, see [2].

In this contribution we are interested in the derivation of new $L^{\infty}$ estimates and we investigate the regularity of the solutions of (1.1). Quite surprisingly, the question of global boundedness becomes trivial when the diffusion coefficients vanish. Indeed, consider $D_{i}=0$, and a bounded initial value. The property (1.4) implies that for each $x$ fixed, the total mass $\sum_{i=1}^{p} m_{i} a_{i}(t, x)$ is time independent. Then, the non-negativity of the $a_{i}$ 's implies that each $a_{i}$ is uniformly bounded. Conversely, certain reaction diffusion systems might exhibit blow-up phenomena, see e.g. [22, 26], as it is also well known when considering nonlinear heat equations $[15,35]$. Therefore global well-posedness and discussion of smoothing effects - that is gain of regularity of the solution compared to the initial data - is an issue.

Standard techniques can indeed be applied to show the existence of a smooth solution of (1.1) locally in time, with, say, initial data in $L^{1} \cap L^{\infty}(\Omega)$. We sketch in the appendix the basic argument that proves the local existence of a smooth non negative solution. The challenging question consists in extending the result on arbitrarily large time intervals. Roughly speaking, this is due to a lack of estimates since the only natural bounds are provided by the mass conservation (1.4) and the entropy dissipation (1.5). In particular, the mass conservation only provides an estimate of the solution in $L^{1}$ which is not enough for the right hand side $Q_{i}(a)$ to make sense as a distribution! However, by using the sophisticated techniques introduced in $[25,26]$, it has been shown recently in [10] that the solutions of (1.1) in the quadratic case (1.6) are a priori bounded in $L^{2}((0, T) \times \Omega)$ so that the nonlinear reaction term makes sense at least in $L^{1}$. This non-trivial estimate can be obtained by exploiting 
the entropy dissipation and the non-degeneracy of the diffusion coefficients. In [10], using also the arguments introduced in [25], it allows us to establish the global existence of weak solutions of (1.1), (1.6). Dealing with higher order nonlinearities or degenerate coefficients the difficulty might lead us to introduce a suitable notion of renormalized solutions, see [10] again. We also mention the recent work [27] where the quadratic system is analyzed with diffusion acting only in one direction. The dissipation property (1.5) is also the basis for studying the asymptotic trend to equilibrium $[8,9]$ in the spirit of the entropy/entropy dissipation techniques which are presented e.g. in [34] (we refer also to [1] for further investigation of the large time behavior of nonlinear evolution systems using the entropy dissipation).

Our approach is inspired by De Giorgi's methods for studying the regularity of solution of diffusion equations without requiring the regularity of the coefficients, see [7]. The crucial step consists in establishing a $L^{\infty}$ estimate on the solution. Regularity of the solution follows in a classical way (see appendix). This approach has been used in [33] to obtain an alternative proof to the regularity results for the Navier-Stokes equation [4, 17] and it also shares some features with the strategy introduced in [29,30]. It has also been applied to study convectiondiffusion equations [18] and regularity for the quasi-geostrophic equation [5]. Here, it is worth pointing out that the proof utilizes strongly the structure of the whole system and the argument is not a mere refinement of a scalar approach. As we shall see however, restrictions appear between the space dimension $N$ and the degree of nonlinearity of the reaction term measured by means of $\bar{\mu}$. For this reason, the $L^{\infty}$ estimates can be proved in two dimension for the quadratic operator (1.6) or in one dimension considering cubic terms.

Theorem 1.1. - We consider the quadratic operator (1.6) (or assume $\bar{\mu}=2$ ). Let $N=2$ and suppose that the diffusion coefficients fulfill (1.2). Let $a_{i}^{0} \geq 0$ satisfy

$$
\sum_{i=1}^{4} \int_{\Omega} a_{i}^{0}\left(1+|x|+\left|\ln \left(a_{i}^{0}\right)\right|\right) \mathrm{d} x=M_{0}<\infty .
$$

Then, (1.1) admits a global solution such that for any $0<T \leq T^{\star}<\infty$, $a_{i}$ belongs to $L^{\infty}\left(\left(T, T^{\star}\right) \times \Omega\right)$.

THEOREM 1.2. - Let $N=1$ with $\bar{\mu} \leq 3$ and suppose that the diffusion coefficients fulfill (1.2). Let $a_{i}^{0} \geq 0$ satisfy (1.7). Then, (1.1) admits a global solution such that for any $0<T \leq T^{\star}<\infty$, a $a_{i}$ belongs to $L^{\infty}\left(\left(T, T^{\star}\right) \times \Omega\right)$.

We point out that these statements do not require any regularity property on the diffusion coefficients $D_{i}$ which are only supposed to be bounded. As a byproduct, by using the new bound, a direct bootstrap argument shows the global regularity of the solution (see appendix).

COROLlary 1.1. - Let the assumptions of Theorem 1.1 or 1.2 be fulfilled. Suppose moreover that the $D_{i}$ 's belong to $C^{k}(\Omega)$ with bounded derivatives up to order $k$. Then, for any $0<T \leq T^{\star}<\infty$, the solution belongs to $L^{\infty}\left(T, T^{\star} ; C^{k}(\Omega)\right)$. Accordingly for $C^{\infty}$ coefficients with bounded derivatives, the solution is $C^{\infty}$ on $\left(T, T^{\star}\right) \times \Omega$.

$4^{\mathrm{e}}$ SÉRIE - TOME $43-2010-\mathrm{N}^{\mathrm{O}} 1$ 
Such statements could be helpful for investigating the large time behavior: they can be the starting point to apply the strategy developed in $[8,9]$ and then this would lead to the proof of the convergence to the equilibrium state for large time, with an exponential rate. We do not discuss further this issue which requires a sharp estimate of the bound with respect to the final time $T^{\star}$. Instead, we consider the case of higher dimensions: the same method provides information on the Hausdorff dimension (definitions are recalled in Section 4) of the set of the singular points of the solutions.

THeOREM 1.3. - Let $N \geq 3$ and $\bar{\mu}=2$. We suppose that the coefficients $D_{i}$ are constant with respect to $x \in \Omega$. Let $a_{i}^{0} \geq 0$ satisfy (1.7). We consider a solution of $(1.1)$ on $(0, T) \times \Omega$. We call a singular point, any point $(t, x)$ having a neighborhood on which one of the function $a_{i}$ is not $C^{\infty}$. Then, the Hausdorff dimension of the set of singular points of the solution a does not exceed $\left(N^{2}-4\right) / N$.

In the next section, we briefly recall the fundamental estimate that follows from (1.5). This bound is used in Section 3 where we adapt De Giorgi's approach to the system (1.1). Section 4 is devoted to the estimate of the Hausdorff dimension of the set of singularities in higher space dimensions.

\section{Entropy dissipation}

In the following sections, we adopt the viewpoint of discussing a priori estimates formally satisfied by the solutions of (1.1). As usual the derivation of such estimates relies on various manipulations such as integrations by parts, permutations of integrals and so on. Of course, such formulae apply to the smooth solutions of the problem that can be shown to exist on a small enough time interval by using classical reasoning for nonlinear parabolic equations (see the appendix). Moreover, these estimates also apply to solutions of suitable approximations of the problem (1.1). Such approximations should be defined so that the essential features of the system are preserved. Hence, let us reproduce the reasoning in [10]: by truncation and regularization we deal with an initial data

$$
a_{i}^{0, \eta} \in C_{c}^{\infty}(\Omega), \quad a_{i}^{0, \eta} \geq 0
$$

which converges in $L^{1}(\Omega)$ to $a_{i}^{0}$ as $\eta>0$ tends to 0 and such that

$\sup _{\eta>0} \sum_{i=1}^{p} \int_{\Omega} a_{i}^{0, \eta}\left(1+|x|+\left|\ln \left(a_{i}^{0, \eta}\right)\right|\right) \mathrm{d} x \leq C_{0} \sum_{i=1}^{p} \int_{\Omega} a_{i}^{0}\left(1+|x|+\left|\ln \left(a_{i}^{0}\right)\right|\right) \mathrm{d} x=C_{0} M_{0}<\infty$.

Next, let us consider a cut-off function $\zeta \in C_{c}^{\infty}(\mathbb{R})$ such that $0 \leq \zeta(s) \leq 1, \operatorname{supp}(\zeta) \subset B(0,2)$ and $\zeta(s)=1$ for $|s| \leq 1$. Then, in (1.1) we replace $Q_{i}(a)$ by

$$
Q_{i}^{\eta}(a)=Q_{i}(a) \zeta(\eta|a|),
$$

with $|a|=\sqrt{a_{1}^{2}+\ldots+a_{p}^{2}}$. Accordingly, for any $\eta>0$ fixed, and $a_{i} \in L^{1}(\Omega), Q_{i}^{\eta}(a)$ belongs to $L^{\infty}(\Omega)$. We can show that the corresponding regularized problem admits a unique (non-negative) smooth solution, globally defined, see [16, 28]. Therefore, in what follows we discuss a priori estimates on solutions of (1.1): for the sake of simplicity we detail the arguments working directly on (1.1), but we keep in mind that the arguments apply to the regularized problem as well. In turn, we obtain bounds on the sequence $a_{i}^{\eta}$, which are 
uniform with respect to $\eta>0$. Finally, existence of a global solution satisfying the estimates follows by performing the passage to the limit $\eta \rightarrow 0$; a detail that we skip here, referring for instance to [10].

We start by discussing the a priori estimates that can be naturally deduced from (1.4) and (1.5). The results here apply in full generality, without assumptions on $p, N, \bar{\mu}$.

Proposition 2.1. - Assume (1.2), (1.4) and (1.5). Let $a_{i}^{0} \geq 0$ satisfy

$$
\sum_{i=1}^{p} \int_{\Omega} a_{i}^{0}\left(1+|x|+\left|\ln \left(a_{i}^{0}\right)\right|\right) \mathrm{d} x=M_{0}<\infty .
$$

We set

$$
\mathfrak{D}(t, x)=\left(\prod_{i=1}^{p} a_{i}^{\mu_{i}}-\prod_{i=1}^{p} a_{i}^{\nu_{i}}\right) \ln \left(\frac{\prod_{i=1}^{p} a_{i}^{\mu_{i}}}{\prod_{i=1}^{p} a_{i}^{\nu_{i}}}\right)(t, x) \geq 0
$$

Then, for any $0<T<\infty$, there exists $0<C(T)<\infty$ such that

$$
\begin{aligned}
\sup _{0 \leq t \leq T}\left\{\sum_{i=1}^{p} \int_{\Omega} a_{i}(1\right. & \left.+|x|+\left|\ln \left(a_{i}\right)\right|\right)(t, x) \mathrm{d} x \\
& \left.+\sum_{i=1}^{p} \int_{0}^{t} \int_{\Omega}\left|\nabla \sqrt{a_{i}}\right|^{2}(s, x) \mathrm{d} x \mathrm{~d} s+\int_{0}^{t} \int_{\Omega} \mathfrak{D}(s, x) \mathrm{d} x \mathrm{~d} s\right\} \leq C(T) .
\end{aligned}
$$

If $\Omega$ is a bounded domain, this estimate holds for $T=+\infty$.

Proof. - As a consequence of (1.4) and (1.5), we get

$$
\frac{\mathrm{d}}{\mathrm{d} t} \sum_{i=1}^{p} \int_{\Omega} a_{i}\left(1+\ln \left(a_{i}\right)\right) \mathrm{d} x+\sum_{i=1}^{p} \int_{\Omega} D_{i} \nabla a_{i} \cdot \frac{\nabla a_{i}}{a_{i}} \mathrm{~d} x+\int_{\Omega} \mathfrak{D} \mathrm{d} x=0 .
$$

Then, the coercivity condition (1.2) means that we can establish the following lower bound

$$
\sum_{i=1}^{p} \int_{\Omega} D_{i} \nabla a_{i} \cdot \frac{\nabla a_{i}}{a_{i}} \mathrm{~d} x \geq \alpha \sum_{i=1}^{p} \int_{\Omega} \frac{\left|\nabla a_{i}\right|^{2}}{a_{i}} \mathrm{~d} x=4 \alpha \sum_{i=1}^{p} \int_{\Omega}\left|\nabla \sqrt{a_{i}}\right|^{2} \mathrm{~d} x .
$$

In the case when $\Omega$ is a bounded domain then the conclusion of the theorem follows as

$$
\begin{aligned}
\sum_{i=1}^{p} \int_{\Omega} a_{i}\left|\ln \left(a_{i}\right)\right| \mathrm{d} x & =\sum_{i=1}^{p} \int_{\Omega} a_{i} \ln \left(a_{i}\right) \mathrm{d} x-2 \sum_{i=1}^{p} \int_{\Omega} a_{i} \ln \left(a_{i}\right) \mathbb{1}_{0 \leq a_{i} \leq 1} \mathrm{~d} x \\
& \leq \sum_{i=1}^{p} \int_{\Omega} a_{i} \ln \left(a_{i}\right) \mathrm{d} x+p \frac{2}{e}|\Omega|,
\end{aligned}
$$

where here and below, $\mathbb{1}_{\mathcal{M}}$ denotes the characteristic function of the set $\mathcal{M}$. In the case when $\Omega=\mathbb{R}^{N}$, then the argument proceeds as follows. By using (1.4) and denoting by $M$ the 
supremum norm of the diffusion coefficients, we get

$$
\begin{aligned}
\frac{\mathrm{d}}{\mathrm{d} t} \sum_{i=1}^{p} \int_{\Omega} a_{i}|x| \mathrm{d} x & =-\sum_{i=1}^{p} \int_{\Omega} D_{i} \nabla a_{i} \cdot \frac{x}{|x|} \mathrm{d} x \\
& \leq M \sum_{i=1}^{p} \int_{\Omega}\left|\nabla a_{i}\right| \mathrm{d} x=M \sum_{i=1}^{p} \int_{\Omega} \frac{\left|\nabla a_{i}\right|}{\sqrt{a_{i}}} \sqrt{a_{i}} \mathrm{~d} x \\
& \leq \frac{\alpha}{2} \sum_{i=1}^{p} \int_{\Omega} \frac{\left|\nabla a_{i}\right|^{2}}{a_{i}} \mathrm{~d} x+\frac{M^{2}}{2 \alpha} \sum_{i=1}^{p} \int_{\Omega} a_{i} \mathrm{~d} x
\end{aligned}
$$

by using the standard inequality $|r s| \leq r^{2} / 2+s^{2} / 2$. Hence, we arrive at

$$
\begin{aligned}
\sum_{i=1}^{p} \int_{\Omega} & a_{i}\left(1+|x|+\ln \left(a_{i}\right)\right) \mathrm{d} x+\frac{\alpha}{2} \sum_{i=1}^{p} \int_{0}^{t} \int_{\Omega} \frac{\left|\nabla a_{i}\right|^{2}}{a_{i}} \mathrm{~d} x \mathrm{~d} s+\int_{0}^{t} \int_{\Omega} \mathfrak{D} \mathrm{d} x \mathrm{~d} s \\
& \leq M_{0}+\frac{M^{2}}{2 \alpha} \sum_{i=1}^{p} \int_{0}^{t} \int_{\Omega} a_{i} \mathrm{~d} x \mathrm{~d} s \\
& \leq\left(1+t M^{2} /(2 \alpha)\right) M_{0}
\end{aligned}
$$

It remains to control the negative part of the $a_{i} \ln \left(a_{i}\right)^{\prime}$ 's. To this end, we use the following classical argument:

$$
\begin{aligned}
\int_{\Omega} a_{i}\left|\ln \left(a_{i}\right)\right| \mathrm{d} x & =\int_{\Omega} a_{i} \ln \left(a_{i}\right) \mathrm{d} x-2 \int_{\Omega} a_{i} \ln \left(a_{i}\right)\left(\mathbb{1}_{0 \leq a_{i} \leq e^{-|x| / 2}}+\mathbb{1}_{e^{-|x| / 2} \leq a_{i} \leq 1}\right) \mathrm{d} x \\
& \leq \int_{\Omega} a_{i} \ln \left(a_{i}\right) \mathrm{d} x+\frac{4}{e} \int_{\Omega} e^{-|x| / 4} \mathrm{~d} x+\int_{\Omega}|x| a_{i} \mathrm{~d} x
\end{aligned}
$$

since $-s \ln (s) \leq \frac{2}{e} \sqrt{s}$ for any $0 \leq s \leq 1$. We conclude by combining together all the pieces.

\section{3. $L^{\infty}$ bounds}

In the spirit of the Stampacchia cut-off method, $L^{\infty}$ bounds of solutions of certain PDEs can be deduced from the behavior of suitable nonlinear functionals. Here, such a functional is constructed in a way that uses the dissipation property (1.5). Let us consider the nonnegative, $C^{1}$ and convex function

$$
\Phi(z)= \begin{cases}(1+z) \ln (1+z)-z & \text { if } z \geq 0 \\ 0 & \text { if } z \leq 0\end{cases}
$$

Then, for $k \geq 0$, we are interested in the evolution of

$$
\sum_{i=1}^{p} \int_{\Omega} \Phi\left(a_{i}-k\right) \mathrm{d} x .
$$


Lemma 3.1. - There exists a universal constant $C$, such that for every $a=\left(a_{1}, \ldots, a_{p}\right)$ solution of (1.1), for any $k \geq 0$, and for any $0 \leq s \leq t<\infty$, we have

$$
\begin{aligned}
\sum_{i=1}^{p} \int_{\Omega} \Phi & \left(a_{i}-k\right)(t, x) \mathrm{d} x+4 \alpha \sum_{i=1}^{p} \int_{s}^{t} \int_{\Omega}\left|\nabla \sqrt{1+\left[a_{i}-k\right]_{+}}\right|^{2}(\tau, x) \mathrm{d} x \mathrm{~d} \tau \\
\leq & \sum_{i=1}^{p} \int_{\Omega} \Phi\left(a_{i}-k\right)(s, x) \mathrm{d} x \\
& +C \sum_{i=1}^{p} \int_{s}^{t} \int_{\Omega}\left(1+k^{\bar{\mu}}+(1+k)\left[a_{i}-k\right]_{+}^{\bar{\mu}-1}\right) \ln \left(1+\left[a_{i}-k\right]_{+}\right)(\tau, x) \mathrm{d} x \mathrm{~d} \tau
\end{aligned}
$$

where $[z]_{+}=\max (0, z)$ denotes the non-negative part of $z$.

Remark 3.1. - Notice that the universal constant does not depend on the actual solution a nor on $k$. It is also worth noticing that, in order to make sense of this inequality we need only $a_{i}^{\bar{\mu}-1} \ln \left(1+a_{i}\right)$ to be integrable, although it is required to have $a_{i}^{\bar{\mu}}$ to be integrable to make sense of the equation (1.1). This point will be very important in the next section. Crucial to the analysis is the similarity of the function $\Phi$ and the natural entropy of the system (1.1).

Proof. - Multiplying (1.1) by $\Phi^{\prime}\left(a_{i}-k\right)$ and summing yield

$$
\frac{\mathrm{d}}{\mathrm{d} t} \sum_{i=1}^{p} \int_{\Omega} \Phi\left(a_{i}-k\right) \mathrm{d} x+\sum_{i=1}^{p} \int_{\Omega} D_{i} \nabla a_{i} \cdot \nabla a_{i} \Phi^{\prime \prime}\left(a_{i}-k\right) \mathrm{d} x=\sum_{i=1}^{p} \int_{\Omega} Q_{i}(a) \Phi^{\prime}\left(a_{i}-k\right) \mathrm{d} x .
$$

Then, we observe that (1.2) leads to

$$
\begin{aligned}
\sum_{i=1}^{p} \int_{\Omega} D_{i} \nabla a_{i} \cdot \nabla a_{i} \Phi^{\prime \prime}\left(a_{i}-k\right) \mathrm{d} x=\sum_{i=1}^{p} \int_{\Omega} D_{i} \nabla a_{i} \cdot \nabla a_{i} \frac{\mathbb{1}_{a_{i} \geq k}}{1+\left[a_{i}-k\right]_{+}} \mathrm{d} x \\
\quad=\sum_{i=1}^{p} \int_{\Omega} D_{i} \nabla\left(1+\left[a_{i}-k\right]_{+}\right) \cdot \nabla\left(1+\left[a_{i}-k\right]_{+}\right) \frac{\mathrm{d} x}{1+\left[a_{i}-k\right]_{+}} \\
\geq \alpha \sum_{i=1}^{p} \int_{\Omega} \frac{\left|\nabla\left(1+\left[a_{i}-k\right]_{+}\right)\right|^{2}}{1+\left[a_{i}-k\right]_{+}} \mathrm{d} x \\
\geq 4 \alpha \sum_{i=1}^{p} \int_{\Omega}\left|\nabla \sqrt{1+\left[a_{i}-k\right]_{+}}\right|^{2} d x .
\end{aligned}
$$

Next, we rewrite the right hand side of (3.1) as

$$
\begin{aligned}
\sum_{i=1}^{p} \int_{\Omega} Q_{i}(a) \ln \left(1+\left[a_{i}-k\right]_{+}\right) \mathrm{d} x \\
=\sum_{i=1}^{p} \int_{\Omega}\left(Q_{i}(a)-Q_{i}\left(1+[a-k]_{+}\right)\right) \ln \left(1+\left[a_{i}-k\right]_{+}\right) \mathrm{d} x \\
\quad+\sum_{i=1}^{p} \int_{\Omega} Q_{i}\left(1+[a-k]_{+}\right) \ln \left(1+\left[a_{i}-k\right]_{+}\right) \mathrm{d} x
\end{aligned}
$$

where (1.5) implies that the last term is non-positive. We are thus left with the task of estimating $\left(Q_{i}(a)-Q_{i}\left(1+[a-k]_{+}\right)\right) \ln \left(1+\left[a_{i}-k\right]_{+}\right)$. 
To this end, let us consider the polynomial function $P: \mathbb{R}^{p} \rightarrow \mathbb{R}$ defined by $P(u)=\prod_{i=1}^{p} u_{i}^{\nu_{i}}$. Clearly, given $u, v \in \mathbb{R}^{p}$, we have

$|P(u)-P(v)|=\left|\int_{0}^{1} \nabla P(u+s(v-u)) \cdot(u-v) \mathrm{d} s\right| \leq C\|u-v\| \int_{0}^{1}\|\nabla P(u+s(v-u))\| \mathrm{d} s$

where $\|\cdot\|$ represents any norm on $\mathbb{R}^{p}$. As a matter of fact, since the $\mu_{i}$ 's and $\nu_{i}$ 's are nonzero integers, we have $\partial_{j} P(u)=\nu_{j} \prod_{i=1}^{p} u_{i}^{\nu_{i, j}^{\prime}}$ where $\nu_{i, j}^{\prime}=\nu_{i}$ if $i \neq j$ and $\nu_{j, j}^{\prime}=\nu_{j}-1$. In particular, note that $\sum_{i=1}^{p} \nu_{i, j}^{\prime}=\bar{\mu}-1$. Therefore, working with the $\ell^{1}$ norm, we get

$$
\|\nabla P(u)\| \leq \sum_{j=1}^{p}\left(\nu_{j} \prod_{i=1}^{p}\left|u_{i}\right|^{\nu_{i, j}^{\prime}}\right)
$$

which yields, by using the convexity of the functions $z \mapsto z^{\nu_{i, j}^{\prime}}$,

$$
|P(u)-P(v)| \leq \sum_{\ell=1}^{p}\left|u_{\ell}-v_{\ell}\right| \times \sum_{j=1}^{p} \nu_{j}\left(\prod_{i=1}^{p}\left|u_{i}\right|^{\nu_{i, j}^{\prime}}+\prod_{i=1}^{p}\left|v_{i}\right|^{\nu_{i, j}^{\prime}}\right) .
$$

Clearly, we have $\prod_{i=1}^{p}\left|u_{i}\right|^{\nu_{i, j}^{\prime}} \leq C \sum_{i=1}^{p}\left(1+\left|u_{i}\right|^{\bar{\mu}-1}\right)$ and finally we obtain

$$
|P(u)-P(v)| \leq C \sum_{\ell=1}^{p}\left|u_{\ell}-v_{\ell}\right| \times \sum_{i, j=1}^{p} \nu_{j}\left(1+\left|u_{i}\right|^{\bar{\mu}-1}+\left|v_{i}\right|^{\bar{\mu}-1}\right) .
$$

We apply this inequality with $u_{i}=a_{i}$ and $v_{i}=1+\left[a_{i}-k\right]_{+}$and we make use of the following simple remarks

$$
\left\{\begin{array}{l}
0 \leq\left(1+\left[a_{i}-k\right]_{+}\right)^{\bar{\mu}-1} \leq C\left(1+\left[a_{i}-k\right]_{+}^{\bar{\mu}-1}\right), \\
0 \leq a_{i} \leq\left[a_{i}-k\right]_{+}+k \text { so that } 0 \leq a_{i}^{\bar{\mu}-1} \leq C\left(\left[a_{i}-k\right]_{+}^{\bar{\mu}-1}+k^{\bar{\mu}-1}\right), \\
\left|a_{i}-\left(1+\left[a_{i}-k\right]_{+}\right)\right| \leq 1+\left|a_{i}-\left[a_{i}-k\right]_{+}\right| \leq 1+k .
\end{array}\right.
$$

Applying the same reasoning with $\mu_{i}$ replacing $\nu_{i}$, we arrive at

$$
\left|Q_{i}(a)-Q_{i}\left(1+[a-k]_{+}\right)\right| \leq C(1+k) \sum_{j=1}^{p}\left(1+k^{\bar{\mu}-1}+\left[a_{j}-k\right]_{+}^{\bar{\mu}-1}\right),
$$

where the constant $C$ depends on $\bar{\mu}$ and $p<\infty$. Then, we end the proof by using the simple inequality: for any $u, v \geq 0$,

$$
u^{\bar{\mu}-1} \ln (1+v)+v^{\bar{\mu}-1} \ln (1+u) \leq 2\left(u^{\bar{\mu}-1} \ln (1+u)+v^{\bar{\mu}-1} \ln (1+v)\right) .
$$

(As usual we have adopted the convention to keep the same notation $C$ for a constant that does not depend on the solution, even when the value of the constant might change from one line to the other.)

REMARK 3.2. - We point out that the arguments above do not extend straightforwardly to situations where the unknown a is an infinite sequence, like e.g. for coagulation-fragmentation models (the constant $C$ involves a sum over the reactants). 
Let $0<T<T^{\star}<\infty$ and $0<K<\infty$ be fixed. Set

$$
0<t_{n}=T\left(1-1 / 2^{n}\right)<T<T^{\star}, \quad 0<k_{n}=K\left(1-1 / 2^{n}\right)<K .
$$

Let us denote

$$
\mathcal{U}_{n}=\sup _{t_{n} \leq t \leq T^{*}} \sum_{i=1}^{p} \int_{\Omega} \Phi\left(a_{i}-k_{n}\right)(t, x) \mathrm{d} x+4 \alpha \sum_{i=1}^{p} \int_{t_{n}}^{T^{\star}} \int_{\Omega}\left|\nabla \sqrt{1+\left[a_{i}-k_{n}\right]_{+}}\right|^{2}(\tau, x) \mathrm{d} x \mathrm{~d} \tau .
$$

The aim is to show that, for a suitable choice of $K>0, U_{n}$ tends to 0 as $n \rightarrow \infty$ which will yield the $L^{\infty}$ bound.

We start by making use of Lemma 3.1 with $0 \leq t_{n-1} \leq s \leq t_{n} \leq t \leq T^{\star}$ and we average with respect to $s \in\left(t_{n-1}, t_{n}\right)$. Since $t_{n}-t_{n-1}=T / 2^{n}$, we obtain

$$
\begin{aligned}
& \frac{T}{2^{n}} \sum_{i=1}^{p} \int_{\Omega} \Phi\left(a_{i}-k_{n}\right)(t, x) \mathrm{d} x+4 \alpha \sum_{i=1}^{p} \int_{t_{n-1}}^{t_{n}} \int_{s}^{t} \int_{\Omega}\left|\nabla \sqrt{1+\left[a_{i}-k_{n}\right]_{+}}\right|^{2}(\tau, x) \mathrm{d} x \mathrm{~d} \tau \mathrm{d} s \\
& \quad \leq \sum_{i=1}^{p} \int_{t_{n-1}}^{t_{n}} \int_{\Omega} \Phi\left(a_{i}-k_{n}\right)(s, x) \mathrm{d} x \mathrm{~d} s+C \sum_{i=1}^{p} \int_{t_{n-1}}^{t_{n}} \int_{s}^{t} \int_{\Omega} \Gamma\left(k_{n}, a_{i}-k_{n}\right)(\tau, x) \mathrm{d} x \mathrm{~d} \tau \mathrm{d} s
\end{aligned}
$$

with the short hand notation $\left.\Gamma(k, u)=\left(1+k^{\bar{\mu}}+(1+k)[u]_{+}^{\bar{\mu}-1}\right)\right) \ln \left(1+[u]_{+}\right)$. Since in the integration domain $s \geq t_{n-1}$ and $t \leq T^{\star}$, the last integral can be dominated by

$\sum_{i=1}^{p} \int_{t_{n-1}}^{t_{n}} \int_{t_{n-1}}^{T^{\star}} \int_{\Omega} \Gamma\left(k_{n}, a_{i}-k_{n}\right)(\tau, x) \mathrm{d} x \mathrm{~d} \tau \mathrm{d} s \leq \frac{T}{2^{n}} \sum_{i=1}^{p} \int_{t_{n-1}}^{T^{\star}} \int_{\Omega} \Gamma\left(k_{n}, a_{i}-k_{n}\right)(\tau, x) \mathrm{d} x \mathrm{~d} \tau$.

Similarly $s \leq t_{n}$ leads to the following bound from below

$$
\begin{aligned}
4 \alpha \sum_{i=1}^{p} \int_{t_{n-1}}^{t_{n}} & \int_{s}^{t} \int_{\Omega}\left|\nabla \sqrt{1+\left[a_{i}-k_{n}\right]_{+}}\right|^{2}(\tau, x) \mathrm{d} x \mathrm{~d} \tau \mathrm{d} s \\
& \geq 4 \alpha \sum_{i=1}^{p} \int_{t_{n-1}}^{t_{n}} \int_{t_{n}}^{t} \int_{\Omega}\left|\nabla \sqrt{1+\left[a_{i}-k_{n}\right]_{+}}\right|^{2}(\tau, x) \mathrm{d} x \mathrm{~d} \tau \mathrm{d} s \\
& \geq 4 \alpha \frac{T}{2^{n}} \sum_{i=1}^{p} \int_{t_{n}}^{t} \int_{\Omega}\left|\nabla \sqrt{1+\left[a_{i}-k_{n}\right]_{+}}\right|^{2}(\tau, x) \mathrm{d} x \mathrm{~d} \tau .
\end{aligned}
$$

Hence, for any $t_{n} \leq t \leq T^{\star}$, we have

$$
\begin{aligned}
& \sum_{i=1}^{p} \int_{\Omega} \Phi\left(a_{i}-k_{n}\right)(t, x) \mathrm{d} x+4 \alpha \sum_{i=1}^{p} \int_{t_{n}}^{t} \int_{\Omega}\left|\nabla \sqrt{1+\left[a_{i}-k_{n}\right]_{+}}\right|^{2}(\tau, x) \mathrm{d} x \mathrm{~d} \tau \\
& \quad \leq \frac{2^{n}}{T} \sum_{i=1}^{p} \int_{t_{n-1}}^{t_{n}} \int_{\Omega} \Phi\left(a_{i}-k_{n}\right)(s, x) \mathrm{d} x \mathrm{~d} s+C \sum_{i=1}^{p} \int_{t_{n-1}}^{T^{\star}} \int_{\Omega} \Gamma\left(k_{n}, a_{i}-k_{n}\right)(\tau, x) \mathrm{d} x \mathrm{~d} \tau .
\end{aligned}
$$

Taking the supremum over $t_{n} \leq t \leq T^{\star}$, we obtain

$$
\begin{aligned}
\mathcal{U}_{n} \leq & \frac{2^{n}}{T} \sum_{i=1}^{p} \int_{t_{n-1}}^{t_{n}} \int_{\Omega} \Phi\left(a_{i}-k_{n}\right)(s, x) \mathrm{d} x \mathrm{~d} s \\
& +C \sum_{i=1}^{p} \int_{t_{n-1}}^{T^{\star}} \int_{\Omega}\left(1+k_{n}^{\bar{\mu}}+\left(1+k_{n}\right)\left[a_{i}-k_{n}\right]_{+}^{\bar{\mu}-1}\right) \ln \left(1+\left[a_{i}-k_{n}\right]_{+}\right)(\tau, x) \mathrm{d} x \mathrm{~d} \tau .
\end{aligned}
$$


The crucial step consists now in establishing the following nonlinear estimate, where restrictions on both the space dimension $N$ and $\bar{\mu}$ appear.

Proposition 3.1. - Suppose $N=1$ or $N=2$. There exists a constant $C>0$ (which does not depend on the solution, nor on $\left.T, T^{\star}, K\right)$ such that

$$
u_{n} \leq C\left(1+T^{\star}\right) \mathcal{K}(n, K, T) u_{n-1}^{(N+2) / N}
$$

where

$$
\begin{aligned}
& \mathcal{K}(n, K, T)=\frac{Q_{K}}{T} 2^{n(2 N+4) / N}+\left(1+K^{\bar{\mu}}\right) S_{K} 2^{n(N+4) / N}+(1+K) R_{K} 2^{n((2 N+4) / N-\bar{\mu})} \\
& \text { and } S_{K}=2 \max \left(1 / K^{(N+4) / N}, 1 / K^{(N+2) / N}\right), R_{K}=2 \max \left(1 / K^{(2 N+4) / N-\bar{\mu}}, 1 / K^{2(N+1) / N-\bar{\mu}}\right), \\
& Q_{K}=S_{K}+2 \max \left(1 / K^{4 / N}, 1 / K^{2 / N}\right) .
\end{aligned}
$$

Let us explain how the restrictions on $N$ and $\bar{\mu}$ work. First of all, it will be crucial to remark that $\mathcal{K}(n, K, T)$ is bounded with respect to $K>1$ provided $\bar{\mu} \leq 2(N+1) / N-1=$ $(N+2) / N$ which means $\bar{\mu}=2$ in dimension $N=2$ and $\bar{\mu}=2$ or 3 in dimension $N=1$. Second of all, we go back to Lemma 3.1 and we shall exploit the dissipation term that comes from the diffusion. Indeed, we expect an estimate of $\Phi\left(a_{i}-k\right)$ in $L^{\infty}\left(0, T^{\star} ; L^{1}(\Omega)\right)$ together with an estimate of $\left(1+\left[a_{i}-k\right]_{+}\right)^{-1 / 2} \nabla\left(1+\left[a_{i}-k\right]_{+}\right)$in $L^{2}\left(\left(0, T^{\star}\right) \times \Omega\right)$. Combining these information would lead to $\nabla Z\left(\left[a_{i}-k\right]_{+}\right) \in L^{2}\left(0, T^{\star} ; L^{1}(\Omega)\right)$ where

$$
Z(u)=\int_{0}^{u} \sqrt{\frac{\Phi(z)}{1+z}} \mathrm{~d} z=\int_{0}^{u} \sqrt{\ln (1+z)+\frac{1}{1+z}-1} \mathrm{~d} z .
$$

Let us consider a non-negative function $u$ defined on $\left[T, T^{\star}\right] \times \Omega$ such that $Z(u)$ belongs to $L^{\infty}\left(T, T^{\star} ; L^{1}(\Omega)\right)$ and $\nabla Z(u)$ belongs to $L^{2}\left(T, T^{\star} ; L^{1}(\Omega)\right)$ According to the GagliardoNirenberg-Sobolev inequality (see [3], Th. IX.9, p. 162) the latter implies that

$$
Z(u) \in L^{2}\left(T, T^{\star} ; L^{N /(N-1)}(\Omega)\right) .
$$

We seek a homogeneous Lebesgue space with respect to the variables $t, x$. For $N \leq 2$ we can obtain:

$$
Z(u) \in L^{(N+2) / N}\left(\left(T, T^{\star}\right) \times \Omega\right) .
$$

Indeed, if $N=2$ we have $(N+2) / N=N /(N-1)=2$, and if $N=1$ :

$\int_{T}^{T^{\star}} \int_{\Omega}|v|^{3} \mathrm{~d} x \mathrm{~d} t \leq \int_{T}^{T^{\star}}\|v(t)\|_{L^{1}(\Omega)}\|v(t)\|_{L^{\infty}(\Omega)}^{2} \mathrm{~d} t \leq\|v\|_{L^{\infty}\left(T, T^{\star} ; L^{1}(\Omega)\right)}\|v\|_{L^{2}\left(T, T^{\star} ; L^{\infty}(\Omega)\right)}^{2}$.

Eventually, we aim at comparing $Z(u)^{(N+2) / N}$ to $\psi(u) \ln (1+u)$ where $\psi: \mathbb{R}^{+} \rightarrow$ $\mathbb{R}^{+}$has a polynomial behavior. Specifying the behavior of $\psi$ will induce restrictions on $\bar{\mu}$ that depend on the space dimension. Of course, it suffices to discuss the comparison as $u \rightarrow 0$ and $u \rightarrow \infty$. Since $\ln (1+z)+1 /(1+z)-1 \sim_{z \rightarrow 0} z^{2} / 2$ we first obtain that $Z(u)^{(N+2) / N} \geq u^{(2 N+4) / N} / 8$ for $u \in[0, \delta], \delta>0$ small enough. It follows that $\psi(u) \ln (1+u)$ can indeed be dominated by $Z(u)$ for bounded $u$ 's provided $\psi(u) \sim_{u \rightarrow 0} u^{(N+4) / N}$. Next, there exists $A>0$ such that for $z \geq A$ large enough, we have $\ln (1+z)+1 /(1+z)-1 \geq$ $\frac{1}{2} \ln (1+z)$. Thus, for $u \geq 2 A$ we get

$$
Z(u) \geq \frac{1}{\sqrt{2}} \int_{u / 2}^{u} \sqrt{\ln (1+z)} \mathrm{d} z \geq \frac{1}{2 \sqrt{2}} u \sqrt{\ln (1+u / 2)} \geq C_{1} u \sqrt{\ln (1+u)} .
$$


Hence $Z(u)^{(N+2) / N}$ dominates $\psi(u) \ln (1+u)$ provided $N \leq 2$ and $\psi(u) \sim_{u \rightarrow \infty} u^{(N+2) / N}$. Reasoning the same way, we also prove that there exists $C>0$ such that $Z(u) \leq C \Phi(u)$ holds for any $u \geq 0$. Let us summarize the properties that we need to justify Proposition 3.1.

Lemma 3.2. - Let us set

$$
\psi(u)=u^{(N+4) / N} \mathbb{1}_{0 \leq u \leq 1}+u^{(N+2) / N} \mathbb{1}_{u \geq 1} .
$$

There exists a constant $C>0$ such that

$$
\psi(u) \ln (1+u) \leq C Z(u)^{(N+2) / N}, \quad \text { and } \quad Z(u) \leq C \Phi(u)
$$

holds for any $u \geq 0$. Furthermore, for every non-negative function u defined on $\left[T, T^{\star}\right] \times \Omega$ we have:

$$
\int_{T}^{T^{\star}}\left|\int_{\Omega}\right| \nabla Z(u)|\mathrm{d} x|^{2} \mathrm{~d} \tau \leq \sup _{T \leq \tau \leq T^{\star}}\left(\int_{\Omega} \Phi(u)(\tau, x) \mathrm{d} x\right) \int_{T}^{T^{\star}} \int_{\Omega}|\nabla \sqrt{1+u}|^{2}(\tau, x) \mathrm{d} x \mathrm{~d} \tau .
$$

Proof of Proposition 3.1. - The proof splits into two steps: firstly we modify (3.2) so that, secondly, we can make the dissipation terms appear by appealing to the Gagliardo-Nirenberg inequality.

Step 1. - The first step consists in showing the following inequality:

$$
\mathcal{U}_{n} \leq C \mathcal{K}(n, K, T) \sum_{i=1}^{p} \int_{t_{n-1}}^{T^{\star}} \int_{\Omega} \psi\left(a_{i}-k_{n-1}\right) \ln \left(1+\left[a_{i}-k_{n-1}\right]_{+}\right) \mathrm{d} x \mathrm{~d} \tau,
$$

where the auxiliary function $\psi$ has been introduced in Lemma 3.2. We start by noting that if $a_{i} \geq k_{n} \geq k_{n-1}$, then $\left(a_{i}-k_{n-1}\right) /\left(k_{n}-k_{n-1}\right) \geq 1$. Therefore we can write for any $\alpha, \beta \geq 0$,

$$
\begin{aligned}
\mathbb{1}_{a_{i} \geq k_{n}} & \leq\left(\frac{\left[a_{i}-k_{n-1}\right]_{+}}{k_{n}-k_{n-1}}\right)^{\alpha} \mathbb{1}_{\left\{k_{n} \leq a_{i} \leq 1+k_{n-1}\right\}}+\left(\frac{\left[a_{i}-k_{n-1}\right]_{+}}{k_{n}-k_{n-1}}\right)^{\beta} \mathbb{1}_{a_{i} \geq 1+k_{n-1}} \\
& \leq \frac{2^{n \alpha}}{K^{\alpha}}\left[a_{i}-k_{n-1}\right]_{+}^{\alpha} \mathbb{1}_{0 \leq a_{i}-k_{n-1} \leq 1}+\frac{2^{n \beta}}{K^{\beta}}\left[a_{i}-k_{n-1}\right]_{+}^{\beta} \mathbb{1}_{a_{i}-k_{n-1} \geq 1} .
\end{aligned}
$$

By using these simple estimates with $\alpha=(N+4) / N, \quad \beta=(N+2) / N \quad$ and $\alpha=(N+4) / N-\bar{\mu}+1, \beta=(N+2) / N-\bar{\mu}+1$ respectively (note that in both case $\alpha \geq \beta$ ), we are led to

$$
\left(1+k_{n}^{\bar{\mu}}\right) \ln \left(1+\left[a_{i}-k_{n}\right]_{+}\right) \leq\left(1+K^{\bar{\mu}}\right) 2^{n(N+4) / N} S_{K} \psi\left(a_{i}-k_{n-1}\right) \ln \left(1+\left[a_{i}-k_{n-1}\right]_{+}\right),
$$

and

$$
\begin{aligned}
\left(1+k_{n}\right)\left[a_{i}-k_{n}\right]_{+}^{\bar{\mu}-1} & \ln \left(1+\left[a_{i}-k_{n}\right]_{+}\right) \\
\leq & (1+K) 2^{n((2 N+4) / N-\bar{\mu})} R_{K} \psi\left(a_{i}-k_{n-1}\right) \ln \left(1+\left[a_{i}-k_{n-1}\right]_{+}\right) .
\end{aligned}
$$

Coming back to (3.2) yields

$$
\begin{aligned}
\mathcal{U}_{n} \leq & \frac{2^{n}}{T} \sum_{i=1}^{p} \int_{t_{n-1}}^{t_{n}} \int_{\Omega} \Phi\left(a_{i}-k_{n}\right) \mathrm{d} x \mathrm{~d} s \\
& +C\left(\left(1+K^{\bar{\mu}}\right) S_{K} 2^{n(N+4) / N}+(1+K) R_{K} 2^{n((2 N+4) / N-\bar{\mu})}\right) \\
& \times \sum_{i=1}^{p} \int_{t_{n-1}}^{T^{\star}} \int_{\Omega} \psi\left(a_{i}-k_{n-1}\right) \ln \left(1+\left[a_{i}-k_{n-1}\right]_{+}\right) \mathrm{d} x \mathrm{~d} \tau .
\end{aligned}
$$

$4^{\text {e }}$ SÉRIE - TOME $43-2010-\mathrm{N}^{\mathrm{o}} 1$ 
The first integral in the right hand side can be dominated in a similar way (using $\alpha=4 / N$, $\beta=2 / N)$; precisely, we have

$$
\begin{aligned}
& \frac{2^{n}}{T} \sum_{i=1}^{p} \int_{t_{n-1}}^{t_{n}} \int_{\Omega} \Phi\left(a_{i}-k_{n}\right) \mathrm{d} x \mathrm{~d} s \\
& \quad \leq \frac{2^{n}}{T} \sum_{i=1}^{p} \int_{t_{n-1}}^{t_{n}} \int_{\Omega}\left(1+\left[a_{i}-k_{n}\right]_{+}\right) \ln \left(1+\left[a_{i}-k_{n}\right]_{+}\right) \mathrm{d} x \mathrm{~d} s \\
& \quad \leq \frac{1}{T} 2^{n(2 N+4) / N} Q_{K} \sum_{i=1}^{p} \int_{t_{n-1}}^{T^{\star}} \int_{\Omega} \psi\left(a_{i}-k_{n-1}\right) \ln \left(1+\left[a_{i}-k_{n-1}\right]_{+}\right) \mathrm{d} x \mathrm{~d} s .
\end{aligned}
$$

Therefore, we have proved from (3.2) that (3.3) holds.

Step 2. - Now, we go back to Lemma 3.2 so that (3.3) becomes

$$
\mathcal{U}_{n} \leq C \mathcal{K}(n, K, T) \sum_{i=1}^{p} \int_{t_{n-1}}^{T^{\star}} \int_{\Omega}\left|Z\left(\left[a_{i}-k_{n-1}\right]_{+}\right)\right|^{(N+2) / N} \mathrm{~d} x \mathrm{~d} \tau
$$

Let us distinguish depending on the dimension $N=1$ or $N=2$ how we conclude by using the Gagliardo-Nirenberg-Sobolev inequality.

For $N=2$, using the Gagliardo-Nirenberg-Sobolev inequality and Lemma 3.2, we obtain

$$
\begin{aligned}
\mathcal{U}_{n} \leq C \mathcal{K}(n, K, T) \sum_{i=1}^{p}[ & \int_{t_{n-1}}^{T^{\star}}\left(\int_{\Omega}\left|\nabla Z\left(\left[a_{i}-k_{n-1}\right]_{+}\right)\right| \mathrm{d} x\right)^{2} \mathrm{~d} \tau \\
& \left.+\int_{t_{n-1}}^{T^{\star}}\left(\int_{\Omega} \Phi\left(\left[a_{i}-k_{n-1}\right]_{+}\right) \mathrm{d} x\right)^{2} \mathrm{~d} s\right] .
\end{aligned}
$$

Then, we use the second statement in Lemma 3.2 to obtain

$$
\begin{aligned}
\mathcal{U}_{n} \leq & C \mathcal{K}(n, K, T) \\
\times \sum_{i=1}^{p} & {\left[\left(\sup _{t_{n-1} \leq \tau \leq T^{\star}} \int_{\Omega} \Phi\left(a_{i}-k_{n-1}\right)(\tau) \mathrm{d} x \int_{t_{n-1}}^{T^{\star}} \int_{\Omega}\left|\nabla \sqrt{1+\left[a_{i}-k_{n-1}\right]_{+}}\right|^{2} \mathrm{~d} x \mathrm{~d} \tau\right)\right.} \\
& \left.\quad+\int_{t_{n-1}}^{T^{\star}}\left(\int_{\Omega} \Phi\left(\left[a_{i}-k_{n-1}\right]_{+}\right) \mathrm{d} x\right)^{2} \mathrm{~d} s\right] \\
\leq & C\left(1+T^{\star}\right) \mathcal{K}(n, K, T) \mathcal{U}_{n-1}^{2} .
\end{aligned}
$$


For $N=1$, we proceed as follows

$$
\begin{aligned}
& \mathcal{U}_{n} \leq C \mathcal{K}(n, K, T) \sum_{i=1}^{p} \int_{t_{n-1}}^{T^{\star}}\left(\left\|Z\left(a_{i}-k_{n-1}\right)(t, \cdot)\right\|_{L^{\infty}(\Omega)}^{2} \int_{\Omega} Z\left(a_{i}-k_{n-1}\right)(t, x) \mathrm{d} x\right) \mathrm{d} t \\
& \leq C \mathcal{K}(n, K, T) \sum_{i=1}^{p}\left[\sup _{t_{n-1} \leq t \leq T^{\star}} \int_{\Omega} Z\left(a_{i}-k_{n-1}\right) \mathrm{d} x\right. \\
& \left.\times \int_{t_{n-1}}^{T^{\star}}\left(\int_{\Omega}\left(\left|Z\left(a_{i}-k_{n-1}\right)\right|+\left|\nabla Z\left(a_{i}-k_{n-1}\right)\right|\right) \mathrm{d} x\right)^{2} \mathrm{~d} t\right] \\
& \leq C \mathcal{K}(n, K, T) \sum_{i=1}^{p}\left[2 T^{\star}\left(\sup _{t_{n-1} \leq t \leq T^{\star}} \int_{\Omega} \Phi\left(a_{i}-k_{n-1}\right) \mathrm{d} x\right)^{3}\right. \\
& \left.+\left(\sup _{t_{n-1} \leq t \leq T^{\star}} \int_{\Omega} \Phi\left(a_{i}-k_{n-1}\right) \mathrm{d} x\right)^{2} \int_{t_{n-1}}^{T^{\star}} \int_{\Omega}\left|\nabla \sqrt{1+\left[a_{i}-k_{n-1}\right]_{+}}\right|^{2} \mathrm{~d} x \mathrm{~d} t\right] \\
& \leq C\left(1+T^{\star}\right) \mathcal{K}(n, K, T) \mathcal{U}_{n-1}^{3} .
\end{aligned}
$$

This ends the proof of Proposition 3.1.

Finishing the proof of the $L^{\infty}$ bound needs the following elementary claim.

Lemma 3.3. - Let $\left(\mathcal{V}_{n}\right)_{n \in \mathbb{N}}$ be a sequence verifying

$$
V_{n} \leq M^{n} V_{n-1}^{q}
$$

for some $M>0, q>1$. Then for any $n_{0} \in \mathbb{N}$, there exists $\varepsilon$, such that if $V_{n_{0}}<\varepsilon$, then $\lim _{n \rightarrow \infty} V_{n}=0$.

Proof. - Without loss of generality we suppose $n_{0}=0$. Let us set $W_{n}=\ln \left(V_{n}\right)$. We have

$$
W_{n} \leq n \ln (M)+q W_{n-1}
$$

which yields

$$
W_{n} \leq \ln (M) \sum_{j=0}^{n} q^{n-j} j+q^{n} W_{0} \leq q^{n} \ln \left(M^{1 /\left(q(1-1 / q)^{2}\right)} V_{0}\right)
$$

So, if $V_{0}<M^{-1 /\left(q(1-1 / q)^{2}\right)}, W_{n}$ converges to $-\infty$, and $V_{n}$ converges to 0

Hence, it remains to check that the first term of the iteration can be made small choosing $K$ large enough. Indeed, let us go back to Proposition 3.1. Picking $K>1$, we can summarize the obtained estimate as

$$
\mathcal{U}_{n} \leq C\left(1+T^{\star}\right)(1+1 / T) 2^{n(2 N+4) / N} u_{n-1}^{(N+2) / N} .
$$

The keypoint is to remark that $Q_{K}, K R_{K}$ and $K^{\bar{\mu}} S_{K}$ remain bounded for large $K$ 's so that the constant $C$ above does not depend on $K$. Hence, we apply Lemma 3.3 to $V_{n}=\left(C\left(1+T^{\star}\right)(1+1 / T)\right)^{2 / N} \mathcal{U}_{n}, q=1+2 / N$ and $M=2^{(2 N+4) / N}$. 
Now, let us specialize (3.4) to the case $n=2$; we get (with $C$ which still does not depend on $K$ )

$$
u_{2} \leq C(1+1 / T)\left[\sum_{i=1}^{p} \int_{0}^{T^{\star}}\left(\int_{\Omega}\left|\nabla Z\left(a_{i}-K / 2\right)\right| \mathrm{d} x\right)^{2} \mathrm{~d} t+\sum_{i=1}^{p} \int_{0}^{T^{\star}}\left(\int_{\Omega} \Phi\left(a_{i}-K / 2\right) \mathrm{d} x\right)^{2} \mathrm{~d} t\right]
$$

in dimension $N=2$ and in dimension $N=1$ the same expression is multiplied by the quantity $\sup _{0 \leq t \leq T^{\star}} \sum_{i=1}^{p} \int_{\Omega} \Phi\left(a_{i}-K / 2\right) \mathrm{d} x$. This allows to establish the following statement.

Lemma 3.4. - Let $\epsilon>0$. Then, there exists $K_{\epsilon} \geq 1$ such that for any $K \geq K_{\epsilon}$ we have $U_{2} \leq \epsilon$.

Proof. - The proof reduces to prove that the two integrals in the right hand side of (3.5) tend to 0 as $K \rightarrow+\infty$. As a matter of fact, there exists $C>0$ such that for any $z \geq 0$ we have $(1+z) \ln (1+z) \leq C z(1+|\ln (z)|)$. Furthermore, there exists $C>0$ such that for any $k>1$ and $z \geq 0$, we have

$$
[z-k]_{+}\left(1+\left|\ln \left([z-k]_{+}\right)\right|\right) \leq C z(1+|\ln z|) .
$$

Accordingly, we deduce that $\Phi\left(a_{i}-K / 2\right)$ converges to 0 for a.e $(t, x) \in\left(0, T^{\star}\right) \times \Omega$ as $K$ goes to infinity and it is dominated by $a_{i}\left(1+\left|\ln \left(a_{i}\right)\right|\right)$, which satisfies

$$
\sum_{i=1}^{p} \int_{0}^{T^{\star}}\left(\int_{\Omega} a_{i}\left(1+\left|\ln \left(a_{i}\right)\right|\right) \mathrm{d} x \mid\right)^{2} \mathrm{~d} t<\infty
$$

owing to Proposition 2.1. Applying the Lebesgue dominated convergence theorem then shows that

$$
\lim _{K \rightarrow \infty}\left\{\sum_{i=1}^{p} \int_{0}^{T^{\star}}\left(\int_{\Omega} \Phi\left(a_{i}-K / 2\right) \mathrm{d} x\right)^{2} \mathrm{~d} t\right\}=0 .
$$

Next, we simply write

$$
\nabla Z\left(a_{i}-K / 2\right)=\mathbb{1}_{a_{i} \geq K / 2} \sqrt{\ln \left(1+\left[a_{i}-K / 2\right]_{+}\right)+\frac{1}{1+\left[a_{i}-K / 2\right]_{+}}-1} \nabla a_{i} .
$$

Then, we remark that $z \mapsto \ln (1+z)+1 /(1+z)-1$ is non-decreasing which allows to establish

$\left|\nabla Z\left(a_{i}-K / 2\right)\right| \leq \mathbb{1}_{a_{i} \geq K / 2} \sqrt{\ln \left(1+a_{i}\right)+\frac{1}{1+a_{i}}-1}\left|\nabla a_{i}\right|=\mathbb{1}_{a_{i} \geq K / 2}\left|\nabla Z\left(a_{i}\right)\right| \leq\left|\nabla Z\left(a_{i}\right)\right|$.

Observe that $\mathbb{1}_{a_{i} \geq K / 2}|\nabla Z(u)|$ decreases to 0 as $K \rightarrow \infty$ for a.e $(t, x) \in\left(0, T^{\star}\right) \times \Omega$. Furthermore, Lemma 3.2 yields

$\sum_{i=1}^{p} \int_{0}^{T^{\star}}\left(\int_{\Omega}\left|\nabla Z\left(a_{i}\right)\right| \mathrm{d} x\right)^{2} \mathrm{~d} t \leq \sum_{i=1}^{p} \sup _{0 \leq t \leq T^{\star}} \int_{\Omega} \Phi\left(a_{i}\right) \mathrm{d} x \int_{0}^{T^{\star}} \int_{\Omega}\left|\nabla \sqrt{1+a_{i}}\right|^{2} \mathrm{~d} x \mathrm{~d} t<\infty$ by using the basic estimates in Proposition 2.1 again. We conclude by classical integration theory arguments. 
We can now finish the proof of Theorem 1.1. Let us emphasize the dependence with respect to $K$ by denoting $u_{n}^{(K)}$. We first fix $K$ which makes $u_{2}^{(K)}$ small enough (remark that $K$ is more constrained as $T$ is chosen small) so that we obtain by applying Lemma 3.3

$$
\lim _{n \rightarrow \infty} \mathcal{U}_{n}^{(K)}=0 .
$$

However, we clearly have

$$
u_{n}^{(K)} \geq \frac{1}{T^{\star}-t_{n}} \int_{t_{n}}^{T^{\star}} \int_{\Omega} \Phi\left(a_{i}-k_{n}\right) \mathrm{d} x \mathrm{~d} t \geq 0 .
$$

Letting $n$ go to infinity and applying the Fatou lemma, we deduce that

$$
\frac{1}{T^{\star}-T} \int_{T}^{T^{\star}} \int_{\Omega} \Phi\left(a_{i}-K\right) \mathrm{d} x \mathrm{~d} t=0,
$$

which implies that $0 \leq a_{i}(t, x) \leq K$ for a.e $(t, x) \in\left(T, T^{\star}\right) \times \Omega$.

Remark 3.3. - Since the initial data is required to satisfy (1.7) only and is not supposed to be bounded, it is clearly hopeless to extend Theorem 1.1 with $T=0$. It appears clearly through the factor $1 / T$ which appears in the estimates above.

\section{Hausdorff dimension of the set of singular points}

In this section we study the Hausdorff dimension of the blow-up points of the solutions of (1.1). The derivation of the necessary estimates remains close to the strategy described in the previous section; again a restriction on the degree of nonlinearity appears. It turns out that relevant results can be obtained by this method in dimension $N \geq 3$ with $\bar{\mu}=2$, while we are not able to reach improvements in direction of higher nonlinearities for lower dimensions. For the sake of simplicity, in what follows we assume that the diffusion coefficients $D_{i}$ are constant with respect to the space variable (but they still depend on $i$, otherwise the problem becomes trivial by remarking that $\rho(t, x)=\sum_{i=1}^{p} a_{i}(t, x)$ satisfies the heat equation $\partial_{t} \rho-D \Delta_{x} \rho=0$, with $D$ the common value of the diffusion coefficients). Then, we shall prove Theorem 1.3.

To begin with, let us recall a few definitions about Hausdorff dimension. For a given nonempty set $A \subset \mathbb{R}^{d}, s \geq 0, \delta>0$, we set

$$
\mathcal{H}_{\delta}^{s}(A)=\inf \left\{\frac{\Gamma(1 / 2)^{s}}{2^{s} \Gamma(s / 2+1)} \sum_{i}\left(\operatorname{diam}\left(A_{i}\right)\right)^{s}, A \subset \bigcup_{i} A_{i}, \operatorname{diam}\left(A_{i}\right) \leq \delta\right\},
$$

and then $\mathcal{H}^{s}(A)=\lim _{\delta \rightarrow 0} \mathcal{H}_{\delta}^{s}(A)$. The Hausdorff dimension of $A$ is defined by

$$
\operatorname{dim}_{\mathcal{H}}(A)=\inf \left\{s>0, \mathscr{H}^{s}(A)=0\right\}=\sup \left\{s>0, \mathscr{H}^{s}(A)=+\infty\right\} .
$$

We refer to [12] (p. 171) for more details.

The starting point of the proof is two-fold. Firstly, we use mass conservation and entropy dissipation to control the solution in a certain $L^{p}$ space, identifying the highest exponent $p$ for which such an estimate is possible. Secondly, we remark that the problem admits an invariant scaling. This is the purpose of the following claims.

$4^{\mathrm{e}}$ SÉRIE - TOME $43-2010-\mathrm{N}^{\mathrm{o}} 1$ 
Lemma 4.1. - Let $N>2$ and $\Omega \subset \mathbb{R}^{N}$. There exists $C>0$ such that for any $T>0$ and for any non-negative function $u \in L^{\infty}\left(0, T ; L^{1}(\Omega)\right)$ verifying $\nabla \sqrt{u} \in L^{2}((0, T) \times \Omega)$, we have

$$
\begin{aligned}
& \int_{0}^{T} \int_{\Omega}|u|^{\frac{(N+2)}{N}} \mathrm{~d} x \mathrm{~d} t \\
& \leq C\|u\|_{L^{\infty}\left(0, T ; L^{1}(\Omega)\right)}^{2 / N}\left(T\|u\|_{L^{\infty}\left(0, T ; L^{1}(\Omega)\right)}+\|\nabla \sqrt{u}\|_{L^{2}((0, T) \times \Omega)}^{2}\right) .
\end{aligned}
$$

Next, we introduce the function

$$
\Psi(X)=X \mathbb{1}_{0 \leq X \leq 1}+\sqrt{X} \mathbb{1}_{X \geq 1} .
$$

There exists $C>0$ such that for any $T>0$ and for any non-negative function $u$ verifying $\nabla \sqrt{u} \in L^{2}((0, T) \times \Omega)$, and $\Phi(u) \in L^{\infty}\left(0, T ; L^{1}(\Omega)\right)$ we have

$$
\begin{aligned}
& \int_{0}^{T} \int_{\Omega}|\Psi(u)|^{\frac{2(N+2)}{N}} \mathrm{~d} x \mathrm{~d} t \\
& \quad \leq C\|\Phi(u)\|_{L^{\infty}\left(0, T ; L^{1}(\Omega)\right)}^{2 / N}\left(T\|\Phi(u)\|_{L^{\infty}\left(0, T ; L^{1}(\Omega)\right)}+\|\nabla \sqrt{1+u}\|_{L^{2}((0, T) \times \Omega)}^{2}\right) .
\end{aligned}
$$

We are concerned with weak solutions of (1.1), that is functions $a_{i}$ that verify (1.1) in the sense of distributions, together with the estimates in Proposition 2.1, deduced from the fundamental properties (1.4) and (1.5) of the system. We shall use the fact that the norm $L^{(N+2) / N}$ of such a solution is finite, as a consequence of (4.1). Another important ingredient relies on the invariant scaling of the equation.

Lemma 4.2. - Let a be a solution of (1.1). Let $t_{0}>0$ and $x_{0} \in \Omega$. Then, for any $0<\varepsilon \ll 1$

$$
a_{\varepsilon}(t, x)=\varepsilon^{2 /(\bar{\mu}-1)} a\left(t_{0}+\varepsilon^{2} t, x_{0}+\varepsilon x\right)
$$

satisfies (1.1).

Lemma 4.2 is straightforward. Let us sketch the proof of Lemma 4.1.

Proof of Lemma 4.1. - There exists a constant $C>0$ such that for any $X \geq 0$

$$
\Psi(X) \leq C \sqrt{\Phi(X)}, \quad \Psi(X) \leq C(\sqrt{1+X}-1) .
$$

Moreover, $\Psi$ is a Lipschitzian function verifying

$$
0 \leq \Psi^{\prime}(X) \leq 2 \sqrt{2} \frac{\mathrm{d}}{\mathrm{d} X}(\sqrt{1+X}-1) .
$$

Hence, we get

$$
\begin{aligned}
\|\Psi(u)\|_{L^{\infty}\left(0, T ; L^{2}(\Omega)\right)}^{2} & \leq C\|\Phi(u)\|_{L^{\infty}\left(0, T ; L^{1}(\Omega)\right)}, \\
\|\nabla \Psi(u)\|_{L^{2}\left(0, T ; L^{2}(\Omega)\right)} & \leq C\|\nabla \sqrt{1+u}\|_{L^{2}\left(0, T ; L^{2}(\Omega)\right)} .
\end{aligned}
$$

Since $2<2(N+2) / N<2 N /(N-2)$, the Hölder inequality yields

$$
\int_{0}^{T} \int_{\Omega}|\Psi(u)|^{\frac{2(N+2)}{N}} \mathrm{~d} x \mathrm{~d} t \leq \int_{0}^{T}\left(\int_{\Omega}|\Psi(u)|^{2} \mathrm{~d} x\right)^{2 / N}\left(\int_{\Omega}|\Psi(u)|^{2 N /(N-2)} \mathrm{d} x\right)^{(N-2) / N} \mathrm{~d} t .
$$


Therefore the Sobolev embedding $H^{1}(\Omega) \subset L^{2 N /(N-2)}(\Omega)$ leads to (4.2) since

$$
\begin{aligned}
\int_{0}^{T} \int_{\Omega}|\Psi(u)|^{\frac{2(N+2)}{N}} \mathrm{~d} x \mathrm{~d} t \\
\quad \leq C\left(\sup _{0 \leq t \leq T} \int_{\Omega}|\Psi(u)|^{2} \mathrm{~d} x\right)^{2 / N} \int_{0}^{T} \int_{\Omega}\left(|\Psi(u)|^{2}+|\nabla \Psi(u)|^{2}\right) \mathrm{d} x \mathrm{~d} t . \\
\quad \leq C\|\Phi(u)\|_{L^{\infty}\left(0, T ; L^{1}(\Omega)\right)}^{2 / N}\left(T\|\Phi(u)\|_{L^{\infty}\left(0, T ; L^{1}(\Omega)\right)}+\int_{0}^{T} \int_{\Omega}|\nabla \sqrt{1+u}|^{2} \mathrm{~d} x \mathrm{~d} t\right) .
\end{aligned}
$$

We obtain (4.1) with a similar combination of the Hölder inequality and the Sobolev embedding.

REMARK 4.1. - We shall use the inequality (4.2) with a sequence of balls $B(0,1) \subset \mathcal{B}_{n} \subset B(0,2)$ as space domain. Since the proof of (4.2) involves the Sobolev embedding, the constant $C$ thus depends on the parameter $n$. However, we can estimate it uniformly. Indeed, the Sobolev embedding on $\Omega=B(0,1)$ reads

$$
\left(\int_{B(0,1)}|u(x)|^{2 N /(N-2)} \mathrm{d} x\right)^{(N-2) / N} \leq C_{1}\left(\int_{B(0,1)}|u(x)|^{2} \mathrm{~d} x+\int_{B(0,1)}|\nabla u(x)|^{2} \mathrm{~d} x\right),
$$

with $C_{1}$ the Sobolev constant on $B(0,1)$. We apply it with $u(x)=\lambda^{(N-2) / 2} u(\lambda x), \lambda>0$. By using the change of variable $y=\lambda x$, it follows that

$$
\left(\int_{B(0, \lambda)}|u(y)|^{2 N /(N-2)} \mathrm{d} y\right)^{(N-2) / N} \leq C_{1}\left(\lambda^{-2} \int_{B(0, \lambda)}|u(y)|^{2} \mathrm{~d} y+\int_{B(0, \lambda)}|\nabla u(y)|^{2} \mathrm{~d} y\right) .
$$

Hence, the Sobolev constant on $B(0, \lambda)$ for any $\lambda>1$ is dominated by $2 C_{1}$.

Keeping in mind Lemma 4.2 now we consider solutions of (1.1) that are defined for negative times. Let us set

$$
k_{n}=1-1 / 2^{n}, \quad t_{n}=1+1 / 2^{n} \quad \mathscr{B}_{n}=B\left(0, t_{n}\right), \quad Q_{n}=\left(-t_{n}, 0\right) \times \mathscr{B}_{n} .
$$

Note that $\mathscr{B}_{n} \subset \mathscr{B}_{n-1}$ and $Q_{n} \subset Q_{n-1}$. We introduce a cut-off function

$$
\begin{cases}\zeta_{n}: \mathbb{R}^{N} \rightarrow \mathbb{R}, & 0 \leq \zeta_{n}(x) \leq 1, \\ \zeta_{n}(x)=1 \text { for } x \in \mathscr{B}_{n}, & \zeta_{n}(x)=0 \text { for } x \in \complement \mathcal{B}_{n-1}, \\ \sup _{i, j \in\{1, \ldots, N\}, x \in \mathbb{R}^{N}}\left|\partial_{i j}^{2} \zeta_{n}(x)\right| \leq C 2^{2 n} . & \end{cases}
$$

We define

$$
\mathcal{U}_{n}=\sup _{-t_{n} \leq t \leq 0} \sum_{i=1}^{p} \int_{\mathcal{B}_{n}} \Phi\left(a_{i}-k_{n}\right) \mathrm{d} x+\sum_{i=1}^{p} \iint_{Q_{n}}\left|\nabla \sqrt{1+\left[a_{i}-k_{n}\right]_{+}}\right|^{2} \mathrm{~d} x \mathrm{~d} s .
$$

Multiplying (1.1) by $\zeta_{n}(x) \Phi^{\prime}\left(a_{i}-k_{n}\right)$ we obtain the following localized version of (3.1)

$$
\begin{aligned}
\frac{\mathrm{d}}{\mathrm{d} t} \sum_{i=1}^{p} \int_{\Omega} \Phi\left(a_{i}-k_{n}\right) \zeta_{n} \mathrm{~d} x+\sum_{i=1}^{p} \int_{\Omega} D_{i} \nabla a_{i} \cdot \nabla a_{i} \Phi^{\prime \prime}\left(a_{i}-k_{n}\right) \zeta_{n} \mathrm{~d} x \\
=\sum_{i=1}^{p} \int_{\Omega} Q_{i}(a) \Phi^{\prime}\left(a_{i}-k_{n}\right) \zeta_{n} \mathrm{~d} x+\sum_{i=1}^{p} \int_{\Omega} D_{i}: D^{2} \zeta_{n} \Phi\left(a_{i}-k_{n}\right) \mathrm{d} x,
\end{aligned}
$$


where $D^{2} \zeta_{n}$ stands for the hessian matrix of $\zeta_{n}$ and $A: B=\sum_{k, l=1}^{N} A_{k l} B_{k l}$. Remark that $0 \leq \mathbb{1}_{\mathcal{B}_{n}}(x) \leq \zeta_{n}(x) \leq \zeta_{n-1}(x) \leq 1$ and $\left|\partial_{k l}^{2} \zeta_{n}(x)\right| \leq 2^{2 n} \mathbb{1}_{\mathcal{B}_{n-1}}(x)$. Then, reproducing the proof of Lemma 3.1 and (3.2) we obtain

$$
\begin{aligned}
\mathcal{U}_{n} \leq & C 2^{2 n} \sum_{i=1}^{p} \iint_{Q_{n-1}} \Phi\left(a_{i}-k_{n}\right)(s, x) \mathrm{d} x \mathrm{~d} s \\
& +C \sum_{i=1}^{p} \iint_{Q_{n-1}}\left(1+k_{n}^{\bar{\mu}}+\left(1+k_{n}\right)\left[a_{i}-k_{n}\right]_{+}^{\bar{\mu}-1}\right) \ln \left(1+\left[a_{i}-k_{n}\right]_{+}\right)(\tau, x) \mathrm{d} x \mathrm{~d} \tau .
\end{aligned}
$$

From this relation we are able to establish the following statements.

Proposition 4.1. - Let $N>2$ and $\bar{\mu}=2$. The following relation holds

$$
u_{n} \leq C 2^{4 n(N+1) / N} u_{n-1}^{1+2 / N}
$$

for any $n \geq 1$. Accordingly, if $\mathcal{U}_{1}$ is small enough then $\lim _{n \rightarrow \infty} \mathcal{U}_{n}=0$.

COROLlary 4.1. - There exists a universal constant $\eta_{\star}>0$ such that any solution of (1.1) satisfying

$$
\sum_{i=1}^{p} \int_{-2}^{0} \int_{B(0,2)}\left|a_{i}\right|^{(N+2) / N} \mathrm{~d} x \mathrm{~d} \tau \leq \eta_{\star}
$$

is such that for any $i \in\{1, \ldots, p\}$ we have

$$
0 \leq a_{i}(t, x) \leq 1 \quad \text { a.e. in }(-1,0) \times B(0,1) .
$$

Proof of Proposition 4.1. - There exists $C_{N}>0$ such that

$$
\begin{aligned}
\Phi\left(a_{i}-k_{n}\right) & \leq\left(1+\left[a_{i}-k_{n}\right]_{+}\right) \ln \left(1+\left[a_{i}-k_{n}\right]_{+}\right) \\
& \leq C_{N}\left(\mathbb{1}_{a_{i} \geq k_{n}}+\left|\Psi\left(a_{i}-k_{n}\right)\right|^{\frac{2(N+2)}{N}}\right) .
\end{aligned}
$$

Similarly, assuming $0 \leq \bar{\mu}-1<1+2 / N$, we can find $C_{N, \bar{\mu}}>0$ such that

$$
\left.\left[a_{i}-k_{n}\right]^{\bar{\mu}-1} \ln \left(1+\left[a_{i}-k_{n}\right]_{+}\right) \leq\left. C_{N, \bar{\mu}}\left(\mathbb{1}_{a_{i} \geq k_{n}}+\mid \Psi\left(a_{i}-k_{n}\right)\right]\right|^{\frac{2(N+2)}{N}}\right) .
$$

For $N \geq 2$, this restricts to the case $\bar{\mu}=2$. Together with (4.4), this gives

$$
u_{n} \leq C 2^{2 n} \sum_{i=1}^{p} \iint_{Q_{n-1}}\left(\mathbb{1}_{a_{i} \geq k_{n}}+\left|\Psi\left(a_{i}-k_{n}\right)\right|^{\frac{2(N+2)}{N}}\right) \mathrm{d} x \mathrm{~d} s .
$$

We note that $0 \leq k_{n} \leq 1$, and $k_{n} \geq k_{n-1}$. Consequently we have

$$
0 \leq \Psi\left(a_{i}-k_{n}\right) \leq \Psi\left(a_{i}-k_{n-1}\right) .
$$

Moreover, we remark that

$$
\mathbb{1}_{a_{i} \geq k_{n}}=\mathbb{1}_{1 \geq a_{i}-k_{n-1} \geq k_{n}-k_{n-1}}+\mathbb{1}_{a_{i}-k_{n-1} \geq 1},
$$


with

$$
\begin{aligned}
\mathbb{1}_{1 \geq a_{i}-k_{n-1} \geq k_{n}-k_{n-1}} & \leq\left(\frac{a_{i}-k_{n-1}}{k_{n}-k_{n-1}}\right)^{\frac{2(N+2)}{N}} \mathbb{1}_{0 \leq a_{i}-k_{n-1} \leq 1} \\
& \leq 2^{\frac{2 n(N+2)}{N}}\left(a_{i}-k_{n-1}\right)^{\frac{2(N+2)}{N}} \mathbb{1}_{0 \leq a_{i}-k_{n-1} \leq 1}, \\
\mathbb{1}_{1 \leq a_{i}-k_{n-1}} & \leq\left(a_{i}-k_{n-1}\right)^{\frac{N+2}{N}} \mathbb{1}_{a_{i}-k_{n-1} \geq 1} \\
& \leq 2^{\frac{2 n(N+2)}{N}}\left(a_{i}-k_{n-1}\right)^{\frac{N+2}{N}} \mathbb{1}_{a_{i}-k_{n-1} \geq 1} .
\end{aligned}
$$

Hence, we have

We are thus led to

$$
\mathbb{1}_{a_{i} \geq k_{n}} \leq 2^{\frac{2 n(N+2)}{N}}\left|\Psi\left(a_{i}-k_{n-1}\right)\right|^{\frac{2(N+2)}{N}} .
$$

$$
\mathcal{U}_{n} \leq C 2^{\frac{4 n(N+1)}{N}} \sum_{i=1}^{p} \iint_{Q_{n-1}}\left|\Psi\left(a_{i}-k_{n-1}\right)\right|^{\frac{2(N+2)}{N}} \mathrm{~d} x \mathrm{~d} s .
$$

Finally, applying Lemma 4.1 (see also Remark 4.1) we obtain

$$
U_{n} \leq C 2^{\frac{4 n(N+1)}{N}} u_{n-1}^{1+2 / N} .
$$

Coming back to Lemma 3.3 finishes the proof of Proposition 4.1.

Proof of Corollary 4.1. - We are thus now left with the task of discussing the smallness of $U_{1}$. Note that $\Psi(X) \leq \sqrt{X}$ for all $X>0$. Hence, from (4.5) with $n=1$, we find

$$
U_{1} \leq C 2^{\frac{4(N+1)}{N}} \sum_{i=1}^{p} \iint_{Q_{0}}\left|a_{i}\right|^{\frac{N+2}{N}} \mathrm{~d} x \mathrm{~d} s=C_{N} \sum_{i=1}^{p}\left\|a_{i}\right\|_{L^{(N+2) / N}((-2,0) \times B(0,2))}^{\frac{N+2}{N}} .
$$

Hence, for $\sum_{i=1}^{p}\left\|a_{i}\right\|_{L^{(N+2) / N}((-2,0) \times B(0,2))}^{(N+2) / N}$ small enough, we have $\mathcal{U}_{1}$ small so that $\lim _{n \rightarrow \infty} \mathcal{U}_{n}=0$, by Proposition 4.1. We conclude by reproducing the arguments at the end of the proof of Theorem 1.1. We have

$$
0 \leq \int_{-t_{n}}^{0} \int_{\mathcal{B}_{n}} \Phi\left(a_{i}-k_{n}\right) \mathrm{d} x \mathrm{~d} t \leq \mathcal{U}_{n} .
$$

Hence letting $n$ go to $\infty$ yields, by using the Fatou lemma,

$$
\lim _{n \rightarrow \infty} \int_{-t_{n}}^{0} \int_{\mathcal{B}_{n}} \Phi\left(a_{i}-k_{n}\right) \mathrm{d} x \mathrm{~d} t=0=\int_{-1}^{0} \int_{B(0,1)} \Phi\left(a_{i}-1\right) \mathrm{d} x \mathrm{~d} t .
$$

It implies that $0 \leq a_{i}(t, x) \leq 1$ holds a. e. on $(-1,0) \times B(0,1)$.

Now, these statements allow us to deduce some property of the solution of the original Cauchy problem. To this end, we go back to the scaling argument in Lemma 4.2. Indeed, we notice that

$$
\begin{aligned}
\int_{-2}^{0} \int_{B(0,2)}\left|a_{\varepsilon}(\tau, x)\right|^{(N+2) / N} \mathrm{~d} x \mathrm{~d} \tau & \\
& =\frac{1}{2} \varepsilon^{2(N+2) / N-(N+2)} \int_{t_{0}-2 \varepsilon^{2}}^{t_{0}+2 \varepsilon^{2}} \int_{\left|y-x_{0}\right| \leq 2 \varepsilon}|a(s, y)|^{(N+2) / N} \mathrm{~d} y \mathrm{~d} s
\end{aligned}
$$


holds (recall that we are dealing with the case $\bar{\mu}=2$ only). We deduce the following statement.

Lemma 4.3. - Let $N \geq 3$ and $\bar{\mu}=2$. Then there exists a universal constant $\eta_{\star}>0$ such that for any a solution of (1.1), any $t_{0}>0, x_{0} \in \Omega$ and $0<\varepsilon \ll 1$, we have the following property. If:

$$
\sum_{i=1}^{p} \frac{1}{\varepsilon^{N+2}} \int_{t_{0}-2 \varepsilon^{2}}^{t_{0}+2 \varepsilon^{2}} \int_{\left|y-x_{0}\right| \leq 2 \varepsilon}|a(s, y)|^{(N+2) / N} \mathrm{~d} y \mathrm{~d} s \leq \eta_{\star} \varepsilon^{-2(N+2) / N}
$$

then $a_{i}$ satisfies $0 \leq a_{i}(t, x) \leq 1 / \varepsilon^{2}$ on $\left|t-t_{0}\right| \leq \varepsilon^{2},\left|x-x_{0}\right| \leq \varepsilon$ and $a_{i}$ is $C^{\infty}$ on this set.

Notice that it is enough to show the boundedness of the $a_{i}$ 's on the neighborhood of $\left(t_{0}, x_{0}\right)$. Then the full regularity on the (possibly smaller) neighborhood is obtained by induction, using classical theory of parabolic equations (see appendix).

We start by localizing: namely, we consider $(0, T) \times B(0, R), 0<T, R<\infty$. We set

$$
\&=\left\{(t, x) \in(0, T) \times B(0, R), a \text { is not } C^{\infty} \text { on a neighborhood of }(t, x)\right\} .
$$

We cover $\phi$ by rectangles with step size $\varepsilon^{2}$ in the time direction and $\varepsilon$ in the space directions, centered at points $(t, x) \in \varnothing$. By the Vitali covering lemma (see [32], p. 9) there exists a countable family denoted by $\left\{C_{j}, j \in \mathbb{N}\right\}$, with $C_{j}$ centered at $\left(t_{j}, x_{j}\right) \in \phi$, made of such rectangles and such that

$$
C_{j} \cap C_{\ell}=\varnothing \quad \text { for } j \neq \ell, \quad \text { and } \quad \phi \subset \bigcup_{j \in \mathbb{N}} \widetilde{C_{j}}
$$

where $\widetilde{C_{j}}$ stands for the rectangle centered at $\left(t_{j}, x_{j}\right)$ with step size $2 \varepsilon^{2}$ in the time direction and $2 \varepsilon$ in the space directions. Since $\left(t_{j}, x_{j}\right)$ does not satisfy the conclusion of Lemma 4.3, we have

$$
\sum_{i=1}^{p} \frac{1}{\varepsilon^{N+2}} \iint_{\widetilde{C}_{j}}\left|a_{i}(s, y)\right|^{(N+2) / N} \mathrm{~d} y \mathrm{~d} s \geq \eta_{\star} \varepsilon^{-2(N+2) / N} .
$$

We introduce the function

$$
F_{\phi}(t, x)=\sum_{j \in \mathbb{N}} \mathbb{1}_{\widetilde{C}_{j}}(t, x) \sum_{i=1}^{p} \frac{1}{\varepsilon^{N+2}} \iint_{\widetilde{C}_{j}}\left|a_{i}(s, y)\right|^{(N+2) / N} \mathrm{~d} y \mathrm{~d} s .
$$

Hence, denoting by $\mathscr{L}$ the Lebesgue measure, we have the following estimate

$$
\begin{aligned}
\mathscr{L}\left(\bigcup_{j \in \mathbb{N}} C_{j}\right) & \leq \mathscr{L}\left(\left\{(t, x) \in(0, T) \times B(0, R), F_{\phi}(t, x) \geq \eta_{\star} / \varepsilon^{2(N+2) / N}\right\}\right) \\
& \leq \frac{\varepsilon^{2(N+2) / N}}{\eta_{\star}} \int_{0}^{T} \int_{\Omega} F_{\phi}(t, x) \mathrm{d} x \mathrm{~d} t
\end{aligned}
$$

as a consequence of the Tchebyschev inequality. It yields by direct evaluation

$$
\begin{aligned}
\mathscr{L}\left(\bigcup_{j \in \mathbb{N}} C_{j}\right) & \leq \frac{\varepsilon^{2(N+2) / N}}{\eta_{\star}} \sum_{i=1}^{p} \sum_{j \in \mathbb{N}}\left(\iint_{\widetilde{C}_{j}}\left|a_{i}\right|^{(N+2) / N} \mathrm{~d} y \mathrm{~d} s \times 2^{N+2} \frac{\int_{0}^{T} \int_{\Omega} \mathbb{1}_{\widetilde{C}_{j}}(t, x) \mathrm{d} x \mathrm{~d} t}{\mathcal{L}\left(\widetilde{C}_{j}\right)}\right) \\
& =2^{N+2} \frac{\varepsilon^{2(N+2) / N}}{\eta_{\star}} \sum_{i=1}^{p}\left\|a_{i}\right\|_{L^{(N+2) / N((0, T) \times \Omega)}}^{(N+2) / N} .
\end{aligned}
$$


Since the Lebesgue measure of the $C_{j}$ 's is proportional to $\varepsilon^{N+2}$, we deduce that the cardinality of the covering is of order $\theta\left(\varepsilon^{2(N+2) / N-(N+2)}=\varepsilon^{-\left(N^{2}-4\right) / N}\right)$. Furthermore, the $\widetilde{C}_{j}$ 's realize a covering of $\phi$ with sets of diameter $\varepsilon$; we conclude that the Hausdorff dimension of $\varnothing$ is dominated by $\left(N^{2}-4\right) / N$.

REMARK 4.2. - It is not obvious that we can improve this estimate, which is in the spirit of [29, 30] for the Navier-Stokes equations, up to a sharp result as in [4, 17]. A difficulty is related to the fact that we are dealing with diffusion coefficients that depend on the component of the system, which prevents from using regularizations by a common heat kernel.

\section{Appendix}

In this appendix, we sketch the proofs of classical results on regularity and small time existence for quasi-linear parabolic systems. The first statements are concerned with the higher regularity of bounded solutions.

Proposition A.1. - Let $T, r>0$ and $x_{0} \in \mathbb{R}^{N}$. Let $u$ be a bounded solution on $[0, T] \times B\left(x_{0}, r\right)$ of

$$
\partial_{t} u-\nabla \cdot(D \nabla u)=f(t, x, u, \nabla u),
$$

with a diffusion matrix $D$ verifying for some $\alpha>0$

$$
D(t, x) \xi \cdot \xi \geq \alpha|\xi|^{2},
$$

for any $(t, x) \in[0, T] \times B\left(x_{0}, r\right)$ and $\xi \in \mathbb{R}^{N}$. The function $f$ lies in $C^{\infty}\left([0, T] \times \mathbb{R}^{N} \times \mathbb{R} \times \mathbb{R}^{N}\right)$ and verifies for any $t \in[0, T], \quad x \in B\left(x_{0}, r\right), \quad|u| \leq M<\infty \quad$ and $p \in \mathbb{R}^{N}$, $|f(t, x, u, p)| \leq C_{M, r}\left(1+|p|^{2}\right)$. Assume in addition that $D \in C^{k}\left([0, T] \times B\left(x_{0}, r\right)\right)$ for an integer $k \geq 1$. Then for every $0<t<T$, we have

$$
u \in L^{\infty}\left(t, T ; C^{k}\left(B\left(x_{0}, r / 2\right)\right) .\right.
$$

In particular, if $D \in C^{\infty}\left([0, T] \times B\left(x_{0}, r\right)\right)$, then for every $t>0$ we have also $u \in C^{\infty}\left([t, T] \times B\left(x_{0}, r / 2\right)\right)$.

This proposition proves Corollary 1.1 from Theorem 1.1 and Theorem 1.2. It is an easy application of the following result (see [16] Theorem 1.1 pp. 419-420 \& Theorem 3.1, pp. 437-438, and, considering systems, Lemma 6.2 p. 592).

Theorem A.1. - Let $T>0$ and $\Omega$ be a bounded domain in $\mathbb{R}^{N}$. Let $D \in C^{1}([0, T] \times \Omega)$ verify the coercivity condition: there exists $\alpha>0$ such that for any $(t, x) \in[0, T] \times \Omega$ and $\xi \in \mathbb{R}^{N}$, we have

$$
D(t, x) \xi \cdot \xi \geq \alpha|\xi|^{2}
$$

Let $M>0$. Let $f \in C^{\infty}\left([0, T] \times \Omega \times[-M, M] \times \mathbb{R}^{N}\right)$ be such that $|f(t, x, u, p)| \leq C_{M}\left(1+|p|^{2}\right)$. Consider a bounded weak solution $u \in L^{\infty}([0, T] \times B),|u(t, x)| \leq M$, to the quasi-linear equation

$$
\partial_{t} u-\nabla \cdot(D \nabla u)=f(t, x, u, \nabla u) .
$$

Then, for any $0<t<T$ and any ball $B$ strictly included in $\Omega, u$ and $\nabla u$ are continuous on $[t, T] \times B$ with $|\nabla u|$ bounded on $[t, T] \times B$. The bound depends only on $t$, the distance of $B$ to $\Omega, M$, the constant $C_{M}$, the coercivity constant $\alpha$, and the Lipschitz norm of $D$.

$4^{\mathrm{e}}$ SÉRIE - TOME $43-2010-\mathrm{N}^{\mathrm{o}} 1$ 
The result of [16] is actually slightly more general (it includes Hölderian regularity of $u$ and $\nabla u$ ), but this statement is enough for our purpose.

Proof of Proposition A.1. - Consider

$$
t_{j}=t\left(1-2^{-j}\right), \quad r_{j}=\frac{r}{2}\left(1+2^{-j}\right) .
$$

We show by induction for $1 \leq j \leq k$ that $u \in L^{\infty}\left(t_{j}, T ; C^{j}\left(B\left(x_{0}, r_{j}\right)\right)\right)$. Theorem A.1 implies that $u \in L^{\infty}\left(t_{1}, T ; C^{1}\left(B\left(x_{0}, r_{1}\right)\right)\right.$. Assume that the result holds for $j \in\{1, \ldots, k-1\}$. Let $\alpha$ be a multi-index in $\mathbb{N}^{N}$ with length $j+1$. Then, $v=\partial^{\alpha} u$ is solution to

$$
\partial_{t} v-\nabla \cdot(D \nabla v)=\tilde{f}(t, x, v, \nabla v)
$$

where the function $\tilde{f}$ verifies the assumption of Theorem A.1, the associated constant $C_{M}$ depending on $t_{j}, r_{j},\|u\|_{L^{\infty}\left(\left[t_{j}, T ; C^{j}\left(B\left(x_{0}, r_{j}\right)\right)\right)\right.}, \alpha$, and $\|D\|_{C^{j+1}}$. Applying Theorem A.1 again gives the estimate with $j+1$.

When $D \in C^{\infty}\left([0, T] \times B\left(x_{0}, r\right)\right)$, once it has been proved that $\partial^{\alpha} u$ is continuous and belongs to $L^{\infty}\left((t, T) \times B\left(x_{0}, r / 2\right)\right)$ for any $\alpha \in \mathbb{N}^{N}$, we establish iteratively the regularity with respect to the time variable.

Next, for the sake of completeness, we give a proof of the existence of smooth and bounded solutions of (1.1) on a small enough time interval.

Proposition A.2. - Let $a^{0} \in\left[L^{\infty}(\Omega)\right]^{p}$ be such that $a_{i}^{0} \geq 0$ for all $i \in\{1, \ldots, p\}$. Then there exist $T_{0}>0$ and $a \in\left[L^{\infty}\left(\left[0, T_{0}\right] \times \Omega\right)\right]^{p}$ solution to (1.1). Moreover this solution is unique, regular on $\left[t, T_{0}\right] \times \Omega$ for any $0<t<T_{0}$ (as long as $D$ is smooth) and verifies $a_{i}(t, x) \geq 0$.

Proof. - Consider $y(t)$ solution to the ODE

$$
\dot{y}=y^{\bar{\mu}}, \quad y(0)=\left\|a^{0}\right\|_{L^{\infty}} .
$$

Let $0<T_{b}<\infty$ be such that

$$
y(t) \leq 2\left\|a^{0}\right\|_{L^{\infty}}, \quad 0 \leq t \leq T_{b} .
$$

Set $a_{i}^{(0)}(t, x)=0$. We construct, by induction for $j \geq 1$, the solutions $a^{(j)}$ on $\left[0, T_{b}\right] \times \Omega$ to the following linear parabolic system

$$
\begin{aligned}
& \partial_{t} a_{i}^{(j)}-\nabla \cdot\left(D_{i} \nabla a_{i}^{(j)}\right)+L_{i}\left(a^{(j-1)}\right) a_{i}^{(j)}=G_{i}\left(a^{(j-1)}\right), \quad i \in\{1, \ldots, p\}, \\
& a_{i}^{(j)}(0, x)=a_{i}^{0}(x),
\end{aligned}
$$

where $L_{i}$ and $G_{i}$ are defined as in (1.3). We show also that $a^{(j)}$ is smooth and verifies

$$
0 \leq a_{i}^{(j)}(t, x) \leq y(t), \quad(t, x) \in\left[0, T_{b}\right] \times \Omega,
$$

and for any $0 \leq t<T_{b}, j \geq 2$

$$
\left\|a^{(j)}(t)-a^{(j-1)}(t)\right\|_{L^{\infty}(\Omega)} \leq 2 p \bar{\mu}\left(2\left\|a^{0}\right\|_{L^{\infty}}\right)^{\bar{\mu}-1} t\left\|a^{(j-1)}-a^{(j-2)}\right\|_{L^{\infty}\left(\left[0, T_{b}\right] \times \Omega\right)} .
$$

Clearly, (A.1) holds for $j=1$ and $j=2$ and (A.2) holds for $j=2$. Assume that we have constructed $a^{(k)}$ for $k \in\{1, \ldots, j\}$ and that (A.1), (A.2) hold for those functions. Note that, for $j$ fixed, the system is decoupled (the definition of $a_{i}^{(j)}$ does not depend on $a_{m}^{(j)}$ for $i \neq m$ ). The existence of a smooth solution $a^{(j+1)}$ follows from the classical theory of linear parabolic 
equations. For $i$ fixed, 0 is a subsolution to the equation satisfied by $a_{i}^{j+1}$ and $y$ is a supersolution. The maximum principle gives the bounds (A.1) for $a^{(j+1)}$. We remark that, for any $i \in\{1, \ldots, p\}$

$$
\begin{aligned}
& \left|G_{i}\left(a^{(j)}\right)-G_{i}\left(a^{(j-1)}\right)\right| \leq 2 \bar{\mu}\left[\sup \left(a^{(j)}, a^{(j-1)}\right)\right]^{\bar{\mu}-1}\left|a^{(j)}-a^{(j-1)}\right|, \\
& \left|L_{i}\left(a^{(j)}\right)-L_{i}\left(a^{(j-1)}\right)\right| \leq 2(\bar{\mu}-1)\left[\sup \left(a^{(j)}, a^{(j-1)}\right)\right]^{\bar{\mu}-2}\left|a^{(j)}-a^{(j-1)}\right| .
\end{aligned}
$$

Moreover, we have

$$
\begin{aligned}
& \quad \partial_{t}\left(a_{i}^{(j+1)}-a_{i}^{(j)}\right)-\nabla \cdot\left(D_{i} \nabla\left(a_{i}^{(j+1)}-a_{i}^{(j)}\right)\right) \\
& \quad=G_{i}\left(a^{(j)}\right)-G_{i}\left(a^{(j-1)}\right)-\left(L_{i}\left(a^{(j)}\right) a^{(j+1)}-L_{i}\left(a^{(j-1)}\right) a^{(j)}\right), \\
& \left(a^{(j+1)}-a^{(j)}\right)(0, x)=0, \quad x \in \Omega .
\end{aligned}
$$

By a comparison principle, we get (A.2) at the rank $j+1$. Hence the induction hypotheses (A.1), (A.2) are satisfied for any $j \geq 2$. Consider $T_{0}=\inf \left(T_{b},\left[4 p \bar{\mu}\left(2\left\|a^{0}\right\|_{L^{\infty}}\right)^{\bar{\mu}-1}\right]^{-1}\right)$. Let $\&$ be the operator defined from $\left[L^{\infty}\left(\left[0, T_{0}\right] \times \Omega\right)\right]^{p}$ to itself by $\phi\left(a^{(j)}\right)=a^{(j+1)}$. Then (A.2) ensures that $\delta$ is a strict contraction. So, by the Banach fixed point theorem, $a^{(j)}$ converges in $L^{\infty}\left(\left[0, T_{0}\right] \times \Omega\right)$ to a function $a$. Passing to the limit in the equation, we get that $a$ is solution to (1.1). Uniform bounds on $a_{i}^{(j)}$ give that $a_{i}$ is non-negative and uniformly bounded by $2\left\|a^{0}\right\|_{L^{\infty}}$ on $\left[0, T_{0}\right] \times \Omega$. Finally, Proposition A.1 proves the regularity of $a$.

\section{REFERENCES}

[1] J. M. BALL, On the asymptotic behavior of generalized processes, with applications to nonlinear evolution equations, J. Differential Equations 27 (1978), 224-265.

[2] M. Bisi, L. Desvillettes, From reactive Boltzmann equations to reaction-diffusion systems, J. Stat. Phys. 124 (2006), 881-912.

[3] H. Brezis, Analyse fonctionnelle. Théorie et applications, Collection Mathématiques Appliquées pour la Maîtrise, Masson, 1983.

[4] L. Caffarelli, R. V. Kohn, L. Nirenberg, Partial regularity of suitable weak solutions of the Navier-Stokes equations, Comm. Pure Appl. Math. 35 (1982), 771831.

[5] L. Caffarelli, A. Vasseur, Drift diffusion equations with fractional diffusion and the quasi-geostrophic equation, to appear in Annals of Math.

[6] J.-F. Collet, Some modelling issues in the theory of fragmentation-coagulation systems, Commun. Math. Sci. 2 (2004), 35-54.

[7] E. De Giorgi, Sulla differenziabilità e l'analiticità delle estremali degli integrali multipli regolari, Mem. Accad. Sci. Torino. Cl. Sci. Fis. Mat. Nat. 3 (1957), 25-43.

[8] L. Desvillettes, K. Fellner, Exponential decay toward equilibrium via entropy methods for reaction-diffusion equations, J. Math. Anal. Appl. 319 (2006), 157-176.

[9] L. Desvillettes, K. Fellner, Entropy methods for reaction-diffusion equations: slowly growing a-priori bounds, Rev. Mat. Iberoam. 24 (2008), 407-431.

[10] L. Desvillettes, K. Fellner, M. Pierre, J. Vovelle, About global existence for quadratic systems of reaction-diffusion, J. Advanced Nonlinear Studies 7 (2007), 491-511.

$4^{\mathrm{e}}$ SÉRIE - TOME $43-2010-\mathrm{N}^{\mathrm{o}} 1$ 
[11] P. Erdi, J. Tóth, Mathematical models of chemical reactions, Nonlinear Science: Theory and Applications, Manchester Univ. Press, 1989.

[12] H. Federer, Geometric measure theory, Die Grund. Math. Wiss., Band 153, Springer New York Inc., New York, 1969.

[13] W. Feng, Coupled system of reaction-diffusion equations and applications in carrier facilitated diffusion, Nonlinear Anal. 17 (1991), 285-311.

[14] P. C. FIFE, Mathematical aspects of reacting and diffusing systems, Lecture Notes in Biomath. 28, Springer, 1979.

[15] Y. Giga, R. V. KoHn, Asymptotically self-similar blow-up of semilinear heat equations, Comm. Pure Appl. Math. 38 (1985), 297-319.

[16] O. A. Ladyzenskaia, V. A. Solonnikov, N. N. Uralceva, Linear and quasi-linear equations of parabolic type, Transl. Math. Monographs 23, AMS, 1968.

[17] F. Lin, A new proof of the Caffarelli-Kohn-Nirenberg theorem, Comm. Pure and Appl. Math. 51 (1998), 241-257.

[18] A. Mellet, A. Vasseur, $L^{p}$ estimates for quantities advected by a compressible flow, J. Math. Anal. Appl. 355 (2009), 548-563.

[19] J. Morgan, Global existence for semilinear parabolic systems, SIAM J. Math. Anal. 20 (1989), 1128-1144.

[20] J. Morgan, Global existence for semilinear parabolic systems via Lyapunov type methods, in Nonlinear semigroups, partial differential equations and attractors (Washington, DC, 1987), Lecture Notes in Math. 1394, Springer, 1989, 117-121.

[21] J. Morgan, Boundedness and decay results for reaction-diffusion systems, SIAM J. Math. Anal. 21 (1990), 1172-1189.

[22] J. Morgan, On a question of blow-up for semilinear parabolic systems, Differential Integral Equations 3 (1990), 973-978.

[23] J. Morgan, S. Waggonner, Global existence for a class of quasilinear reactiondiffusion systems, Commun. Appl. Anal. 8 (2004), 153-166.

[24] J. D. Murray, Mathematical biology, Interdisciplinary Applied Math. 17 \& 18, Springer, 2003.

[25] M. Pierre, Weak solutions and supersolutions in $L^{1}$ for reaction-diffusion systems, $J$. Evol. Equ. 3 (2003), 153-168.

[26] M. Pierre, D. Schmitt, Blowup in reaction-diffusion systems with dissipation of mass, SIAM Rev. 42 (2000), 93-106 (electronic).

[27] M. Pierre, R. Texier-Picard, Global existence for degenerate quadratic reactiondiffusion systems, to appear in Ann. IHP Anal. non linéaire.

[28] F. Rотне, Global solutions of reaction-diffusion systems, Lecture Notes in Math. 102, Springer, 1984.

[29] V. SCHefFer, Partial regularity of solutions to the Navier-Stokes equations, Pacific J. Math. 66 (1976), 535-552.

[30] V. Scheffer, Hausdorff measure and the Navier-Stokes equations, Comm. Math. Phys. 55 (1977), 55-97.

[31] L. W. Somathilake, J. M. J. J. Peiris, Global solutions of a strongly coupled reaction-diffusion system with different diffusion coefficients, J. Appl. Math. 1 (2005), 23-36. 
[32] E. M. Stein, Singular integrals and differentiability properties of functions, Princeton Mathematical Series, No. 30, Princeton Univ. Press, 1970.

[33] A. Vasseur, A new proof of partial regularity of solutions to Navier-Stokes equations, NoDEA Nonlinear Differential Equations Appl. 14 (2007), 753-785.

[34] C. Villani, Hypocoercive diffusion operators, in Proceedings of the International Congress of Mathematicians, 2006.

[35] F. B. Weissler, An $L^{\infty}$ blow-up estimate for a nonlinear heat equation, Comm. Pure Appl. Math. 38 (1985), 291-295.

(Manuscrit reçu le 20 février 2008; accepté, après révision, le 11 mai 2009.)

\author{
Thierry Goudon \\ Project-Team SIMPAF-INRIA Lille Nord Europe \\ \& Labo. Paul Painlevé CNRS-USTLille, \\ Park Plazza, 40 avenue Halley \\ 59650 Villeneuve d'Ascq cedex, France \\ E-mail: thierry.goudon@inria.fr \\ Alexis Vasseur \\ Department of Mathematics \\ University of Texas at Austin \\ Austin, Texas 78712, USA \\ E-mail: vasseur@math.utexas.edu
}

